\title{
A consensus guide to using functional near-infrared spectroscopy in posture and gait research
}

\author{
Jasmine C. Menant ${ }^{a}$, Inbal Maidan ${ }^{b}$, Lisa Alcock ${ }^{c,}$ Emad Al-Yahyad, Antonio Cerasa ${ }^{\text {e }}$, David \\ J. Clark ${ }^{f}$, Eling de Bruin, Sarah Fraser ${ }^{h}$, Vera Gramignai, Dennis Hamacher $^{j}$, Fabian Herold, \\ Roee Holtzer', Meltem Izzetoglum ${ }^{m}$, Shannon Lim ${ }^{n, o}$, Annette Pantall ${ }^{p}$, Paulo Pelicioni ${ }^{a}$, Sue \\ Peters $^{\circ}$, Andrea L. Rosso ${ }^{q}$, Rebecca St George ${ }^{r}$, Samuel Stuart ${ }^{s}$, Roberta Vastai', Rodrigo \\ Vitoriot, Anat Mirelman ${ }^{b}$.
}

a Neuroscience Research Australia, University of New South Wales, New South Wales, Australia; School of Public Health and Community and Medicine, University of New South Wales, New South Wales, Australia.

${ }^{b}$ Laboratory for Early Markers of Neurodegeneration (LEMON), Center for the study of Movement, Cognition, and Mobility (CMCM), Neurological Institute, Tel Aviv Sourasky Medical Center, Israel; Department of Neurology, Sackler School of Medicine and Sagol School of Neuroscience, Tel Aviv University, Tel Aviv, Israel.

C Translational and Clinical Research Institute, Campus for Ageing and Vitality, Newcastle University, Newcastle upon Tyne, United Kingdom.

d Department of Physiotherapy, School of Rehabilitation Sciences, The University of Jordan, Amman, Jordan; Movement Science Group, Faculty of Health and Life Sciences, Oxford Brookes University, Oxford, UK.

e IRIB, National Research Council, Mangone (CS), Italy; S. Anna Institute and Research in Advanced Neurorehabilitation (RAN), Crotone, Italy. 
${ }^{\mathrm{f}}$ Department of Aging and Geriatric Research, University of Florida, Gainesville, FL, USA;

Brain Rehabilitation Research Center, Malcom Randall VA Medical Center, Gainesville, FL, USA.

g Institute of Human Movement Sciences and Sport, Department of Health Sciences and Technology, ETH Zürich, Zurich, Switzerland; Division of Physiotherapy, Department of Neurobiology, Care Sciences and Society, Karolinska Institute, Huddinge, Sweden.

h École interdisciplinaire des sciences de la santé (Interdisciplinary School of Health Sciences), University of Ottawa, Ottawa, Ontario, Canada.

'Neuroscience Research Center, "Magna Graecia” University, Catanzaro, Italy.

jGerman University for Health and Sports, (DHGS), Berlin, Germany.

${ }^{k}$ Research Group Neuroprotection, German Center for Neurodegenerative Diseases (DZNE), Magdeburg, Germany; Department of Neurology, Medical Faculty, Otto von Guericke University, Magdeburg, Germany.

'Yeshiva University, Ferkauf Graduate School of Psychology; The Saul R. Korey Department of Neurology, Albert Einstein College of Medicine, Bronx, NY, USA.

m Villanova University, Electrical and Computer Engineering Department, Villanova, PA, USA

${ }^{\mathrm{n}}$ Graduate Program in Rehabilitation Sciences, University of British Columbia, Vancouver, Canada.

${ }^{\circ}$ Department of Physical Therapy, Faculty of Medicine, University of British Columbia, Vancouver, BC, Canada,.

Rehabilitation Research Program, Vancouver Coastal Health Research Institute, Vancouver, BC, Canada. 
${ }^{\mathrm{p}}$ Translational and Clinical Research Institute, Campus for Ageing and Vitality, Newcastle University, Newcastle upon Tyne, United Kingdom.

a Department of Epidemiology, Graduate School of Public Health, University of Pittsburgh, Pittsburgh, USA.

${ }^{r}$ Sensorimotor Neuroscience and Ageing Research Group, School of Psychological Sciences, College of Health and Medicine, University of Tasmania, Hobart, Australia.

${ }^{s}$ Department of Sport, Exercise and Rehabilitation, Northumbria University, Newcastle upon Tyne, UK.

${ }^{\mathrm{t}}$ Department of Neurology, Oregon Health \& Science University, Portland, Oregon, USA.

$\triangle$ Corresponding author:

Dr Jasmine Menant,

Neuroscience Research Australia,

Barker Street, Randwick, NSW, 2031, Australia.

Email: j.menant@neura.edu.au

Word count: 5780

1 Table, 3 Figures, 1 Table in Supplementary Materials 


\begin{abstract}
Background: Functional near-infrared spectroscopy (fNIRS) is increasingly used in the field of posture and gait to investigate patterns of cortical brain activation while people move freely. fNIRS methods, analysis and reporting of data vary greatly across studies which in turn can limit the replication of research, interpretation of findings and comparison across works.
\end{abstract}

Research question and methods: Considering these issues, we propose a set of practical recommendations for the conduct and reporting of fNIRS studies in posture and gait, acknowledging specific challenges related to clinical groups with posture and gait disorders. Results: Our paper is organized around three main sections: 1) hardware set up and study protocols, 2) artefact removal and data processing and, 3) outcome measures, validity and reliability; it is supplemented with a detailed checklist.

Significance: This paper was written by a core group of members of the International Society for Posture and Gait Research and posture and gait researchers, all experienced in fNIRS research, with the intent of assisting the research community to lead innovative and impactful fNIRS studies in the field of posture and gait, whilst ensuring standardization of research.

Keywords: functional-Near Infrared Spectroscopy; guidelines: cerebral hemodynamics; posture; gait; balance.

Funding: This research did not receive any specific grant from funding agencies in the public, commercial or not-for-profit sectors. 
Declarations of interest: None

\section{Introduction}

Functional near-infrared spectroscopy (fNIRS) is an optical neuroimaging technique that monitors hemodynamic responses in superficial cortical regions. The fNIRS raw data extracted from most devices is light intensity. Through computation of the differential light intensity between the input and output, these data can then be converted to represent changes in the concentration of oxygenated and deoxygenated hemoglobin ( $\mathrm{HbO} 2$ and $\mathrm{HHb}$, respectively) across all vascular compartments (arteries, veins and capillaries) [1]. The neurovascular coupling process enables these $\mathrm{HbO} 2$ and $\mathrm{Hhb}$ concentration changes to be considered as surrogates for neural activation [2-4]. The fNIRS technique has revolutionised the field of posture and gait largely due to its portability; the ability to assess brain activation during actual task performance (i.e., walking, balancing). As such, it addresses a key limitation of other commonly used neuroimaging techniques such as functional magnetic resonance imaging, which involves static tasks and/or supine posture in order to minimize movement.

The increasing availability of commercial fNIRS devices has facilitated the extensive use of this technique to investigate cortical contributions to gait and postural control. fNIRS has been used to explore questions relating to cortical activation during balance tasks (e.g. [5-10]), stepping tasks (e.g. $[6,11])$, walking over unobstructed paths (e.g. $[12,13])$ or paths with obstacles (e.g. [14-17]), treadmill walking (e.g. [18-24]) and walking with and without concurrently performing secondary cognitive (e.g. [12, 25-30]) or motor tasks (e.g. [31]). The majority of studies focused on young and older adults (e.g. [12, 23, 24, 28, 30, 32, 33]), but some research has involved clinical populations (e.g., Parkinson's disease (e.g. [34-41]), stroke 
(e.g. [17, 42-48]), multiple sclerosis (e.g. [49-52]). Areas of interest have primarily covered the prefrontal cortex (e.g. $[12,20,31,53])$, the pre-supplementary motor area (e.g. [20]), the supplementary motor area (e.g. [20,31]), the premotor cortex (e.g. $[6,7,32,33])$, the primary motor cortex (e.g. $[6,7,20])$, the sensorimotor cortex (e.g. $[20,33])$, the superior temporal gyrus (e.g. [5]) and all superficial cortical areas that the near-infrared light can penetrate. The results of the published studies have increased our understanding of the cortical involvement in gait and postural control and can be interpreted in the context of theories relating to neural compensation, inefficiency and capacity [54]. These theories relate to either the increase in neural activation efforts to maintain performance despite declining brain capacity (also known as "less wiring, more firing") [55-57] or the capacity limitation model which suggests that a reduction in activation is synonymous to limited brain resources resulting in poor performance on one or both tasks.

The increasing number of studies using fNIRS in balance and gait research is demonstrated by the rising number of published systematic reviews, $>15$ published in the past 10 years (e.g., [58-72]). Yet from these reviews, it is apparent that the obvious benefits related to knowledge growth are hampered by the inconsistency and lack of details in the reporting of experimental and data analysis protocols. This significantly limits the replication of research, its interpretation in a wider context and comparison across works. Aside from practical points and take-home messages provided in the conclusions of reviews, guidelines regarding the reporting of fNIRS data in posture and gait research do not exist. In view of these concerns, the goal of this consensus paper is to summarize the current state of knowledge on the use of fNIRS for the study of posture and gait and identify knowledge gaps that offer high probability of leading to innovations in the field. The paper is divided into three main sections: 
1) hardware set up and study protocols, 2) artefact removal and data processing and 3) outcome measures, validity and reliability.

\section{Hardware set up and study protocols}

Many different fNIRS devices and configurations have been used in the field of posture and gait, including custom-made and commercially available units. Some systems offer single channels to measure from specific regions of interest (ROIs) while others offer many channels covering broader areas of the scalp, both have advantages and limitations $[73,74]$. Multichannel units present the obvious benefit of recording from more cortical regions in a single recording session, but also suffer from lower sampling rates as a result of signal multiplexing needed to distinguish between channels [73]. This can have an adverse impact on data quality because low sampling rates preclude the ability to apply some of the recommended signal processing steps. Single channels on the other hand focus on a single ROI, which in complex functions such as gait and balance may limit our understanding of the network of regions involved and important changes across regions that may occur with different task demands or in response to interventions. Ultimately, the choice of fNIRS device should be motivated by the specific research questions.

Because of the comparative nature of the fNIRS technique, hemodynamic changes can be explored in an event-related or block design (Figure 1). In both cases, recording needs to be of sufficient duration to observe the onset (about 1-2 seconds after neural firing) and peak (about 4-7 seconds) of the hemodynamic response [75]. Block designs are generally appropriate to measure both transient and sustained cortical activity related to experimental tasks involving prolonged continuous, reciprocal movements. Walking and steady state 
standing are good examples. In block design trials, baseline periods following experimental task periods should be sufficient for the hemodynamic response to return towards its original baseline levels. It is important to consider that for block design paradigms with as little as four repetitions, anticipatory responses may occur [32]. This can be controlled for by varying baseline intervals so that the onset of the experimental task is difficult to predict or use a specific section within the middle of each block. There is currently no gold standard for the number of trials required to reduce variability of fNIRS signal $[61,68,70,72]$. Nevertheless, using at least three trials will allow averaging over several fNIRS signals and should minimize anticipatory contributions.

Event-related designs tend to be more suited to measuring cortical activity in response to acute events, such as gait initiation, postural reactions to balance perturbations, and specific gait phenomena such as freezing of gait, turns or obstacle negotiation (e.g. $[6,11,16,35])$. In such a design, it is crucial to synchronize the event with the fNIRS signals. To capture the hemodynamic response, the protocol should be designed to record at least 3 seconds of the time: before the event, during the event and after the event; this will enable to capture the peak of the response for a single stimulus. For event-related designs, shorter baselines will allow significantly more trials to do more powerful statistics [76]. Conversely, it is also important to consider appropriate inter-stimulus interval which, if too brief, will cause the event-related responses to overlap, in turn compromising the nature of the event-related design. This event-related method allows investigating individual response to a stimulus but poses a challenge when compared within or between groups due to the potential betweensubjects variance in hemodynamic response. It is thus essential for researchers to detail the experimental procedure and account for differences between subjects where applicable. 
These inherent limitations of fNIRS methodology should be considered carefully in protocol design. An emphasis should be placed on selecting an appropriate baseline for the task studied. Since posture and gait studies are conducted upright, baseline fNIRS recordings have to be in upright position to eliminate changes due to gravitational blood pressure fluctuations [77].

\section{Optode placement}

To ensure scientific rigor and reproducibility, optode placement on the scalp should be reported relative to anatomical landmarks. The common approach is to use the international 10-20 system, which defines scalp locations as a percentage of the individual's head size [78]. Initial measurements include mid-sagittal plane distance (nasion to inion), a frontal plane distance (left to right pre-auricular point), and head circumference. Ideally, in the case of customizable optode arrays, specific standardized scalp locations should be determined based on percentages of those initial measurements. Given the obvious ambiguity in localizing surface anatomy landmarks (e.g. peri-auricular points and inion)[79], explicitly defining landmark locations is important for maintaining consistent landmarking optode locations across sessions.

A key concern to any fNIRS research study is to ensure that the optode location effectively
targets the selected underlying cortical ROI. The Gold standard method is to obtain a recent
structural Magnetic Resonance Imaging (MRI) scan of the individual's brain and co-register
the digitized optode locations on the scalp with the underlying cortical site(s). Yet the costs
and logistics associated with brain MRI data collection can be a major obstacle. In the absence
of brain MRI scans, the fNIRS Optodes Location Decider (fOLD) approach and the use of 3D 
digitization are available to guide the selection of optode positions for fNIRS experiments [80]. The fOLD method is based on photon transport simulations on two head atlases and the toolbox is freely available for download (Table S1). The 3D digitizing method allows to project optode locations onto brain atlases [81]. The translation of optodes positioning to precise cortical ROls remains a challenge because there can be considerable variability in brain morphology among individuals. In particular, existing neuroimaging research on brain morphology has identified large variation in older adults and people with brain pathologies such as stroke, traumatic brain injury, or neurodegeneration $[82,83]$. This should be taken into consideration when evaluating between-subject designs.

In within-subjects designs, a convenient way to improve consistency is to supplement $10-20$ land marking with digitization of the optode using a 3D digitizing pen. Differences between optode locations across multiple testing sessions can then be calculated to determine the variance in optode placement [84]. If the estimated optode location has a large difference between sessions (i.e. greater than the inter-optode distance), the following options should be taken: 1) discard the optode from multi-session comparisons, 2) determine if another optode was set up closer to the optode of interest.

\section{Caps, hair, scalp and chinstraps considerations}

Optodes are typically held in place by a cap or headband. Most caps are flexible and often come with pre-cut holes (some corresponding to 10-20 landmarks) hence allowing for customizable optode arrays. However, variation in the relative stretch of the cap over different scalp areas or between participants can alter the inter-optode distance, affect signal intensity, and introduce variability in inter-subject optode locations. 
Optodes with a pointed tip might be required when the desired optode location is covered by hair. However, this might increase noise level relative to the signal. Further, the pointed-tip optode design is likely to increase pressure at optode locations, in order to maximize contact with the scalp. The increased pressure may further impact skin blood flow which can increase superficial layer contamination in fNIRS measurements. The pressure from the optodes may also cause discomfort for the participant. In this situation, the recorded cortical activity could be biased by attention to the discomfort and further limit the tolerable duration of the testing time. Strategies to manage this issue include keeping data collection sessions short and/or taking extra time to separate the hair beneath each optode such that tightening of the cap can be minimized to avoid discomfort for the participant.

If a chinstrap is used to secure the cap in place, it can increase the risk of talking-induced movement artefacts $[85,86]$. This is particularly important for studies that include tasks requiring vocal response, such as in dual-task paradigms that pair walking or balance with a verbal cognitive task. Headband configuration units are less influenced by verbal responses, however, measurements are limited to the prefrontal cortex. In some systems the optode configurations are adjustable while in other they are fixed in place, which limits flexibility of the array but ensures consistent inter-optode distance and improves optode placement uniformity across participants. Differences in brain morphology may influence the signal and interpretation, therefore, they should be reported and taken into consideration during analysis. Future consensus efforts should be made by posture and gait researchers to achieve standardisation of optode positioning through the establishment of brain fNIRS-MRI repositories. 


\section{Artefact removal and data processing}

fNIRS signals are influenced by a variety of confounding factors that should be controlled for to optimize data quality. fNIRS data should be recorded with an adequate signal-to-noise ratio reflected in a close coupling of the optodes with the scalp. A few checks can be used to ensure good data quality prior to data acquisition: (i) heart rate oscillations clearly visible in each channel [87]; (ii) channel-wise metrics set-up by the manufacturers and which rely, for instance, on the calculation of the coefficient of variation to rate signal quality (Table S1); (iii) use of freely available software 'PHOEBE' which detects cardiac pulsation automatically and can be used to adjust and ensure a relative optimal optode-scalp coupling [88]. This section reviews common confounding factors and methodologies used in the posture and gait field to account for them. Figure 2 provides a summary of the fNIRS data processing steps.

\section{Environmental conditions}

The environmental conditions of laboratory settings (e.g. room temperature, humidity, sound, light) should be kept stable to ensure that the electronic devices perform optimally and that the participants do not experience discomfort. For example heat stress would influence the cardiorespiratory system, inducing systemic physiological changes (e.g. increased heart rate and blood flow) which may confound the fNIRS signal and lead to 'false positive' findings $[89,90]$. Sweating is also likely to affect light sources and detector coupling with the skin. Loud sounds could also affect chromophore concentration through attentional interference, as seen in functional MRI experiments [91]. It is also recommended to conduct the experiments in a room with dimmed lights and/or to use a dark head cap to cover and 
shield the optodes from ambient light [89] as light, including variations in colored light, has been found to contaminate signals [92-94].

\section{Instrument-related artefacts}

Instrumental configurations such as wavelength selection, measurement frequency and type of light detectors can influence the signal quality, however, they cannot be easily changed by the user. Hence, the importance of carefully reporting them in sufficient detail and following the manufacturers' instructions. With regard to the illumination source, lasers require some heating time to perform optimally; thus it is recommended that the instrumentation be switched on with some time before starting fNIRS data acquisition [89]. To reduce cross-talk (e.g. incorrect separation of changes in $\mathrm{HbO} 2$ and $\mathrm{HHb}$ ) which heavily depends on the wavelength selection, an optimal combination of wavelengths should be used $[73,89]$. Even though there is currently no consensus as to which combination of wavelengths is optimal $[61,73]$, the degree of cross-talk has been deemed to be relatively minimal when using one wavelength $>730 \mathrm{~nm}$ and another $<720 \mathrm{~nm}$ [95]. Of note, commonly used commercial systems do not allow changing these parameters and typically report one wavelength between $705 \mathrm{~nm}$ and $760 \mathrm{~nm}$ and another around $850 \mathrm{~nm}$ [66].

\section{Motion-related artefacts}

In any balance and gait research, motion-related artefacts are unavoidable because of the movement involved in the execution of balance or walking tasks. Head motion might lead to changes in optode-scalp coupling which in turn, influences light detection [89]. It can further cause changes in the measured cortical location or shifts in cortical hemodynamic levels irrelevant of task related activations. These distinct effects can be reflected as different types 


\section{Physiology-related artefacts}

fNIRS signals not only record changes in cerebral hemodynamics but are also affected by variations in systemic physiology (e.g. fluctuations in heart rate, respiration, and/or blood pressure) [90]. These can increase the risk of finding 'false positives' because detected hemodynamic responses are wrongly attributed to functional brain activity. Thus, in order to elucidate the physiological origin of observed hemodynamic brain changes, it is possible to use multimodal physiological monitoring; an approach which has recently been termed 'systemic-physiology-augmented fNIRS' (SPA-fNIRS) neuroimaging [90, 93, 94]. This method applies short-separation channels to quantify systemic changes in the extracerebral layer [61, 
$70,90]$ and to remove skin response (the overall effect of extracerebral or superficial layers) from the long separation channels to obtain the cortical responses $[90,102,103]$. In addition, it is possible to capture changes in heart rate (e.g. via portable heart rate monitor or a pulse oximeter), blood pressure (e.g. based on pulse transit time), electrodermal activity (e.g. via skin conductance response) and respiration (e.g. via breathing rate and arterial partial pressure of carbon dioxide) $[93,94,104]$; the downside being over-instrumenting participants which may interfere with natural walking patterns.

\section{Post data acquisition processing}

To process and analyze fNIRS data, custom-written scripts, open-source toolboxes [96] or fNIRS manufacturers' software can be used (Table S1). However, regardless of which are utilized, processing information should be reported transparently and with sufficient detail to be replicated.

\section{Visual inspection and motion artefact removal}

As a first step, visual inspection of raw and/or relative optical density data is necessary to get an overview of data quality. Channels with insufficient data quality (see Table S1 for definitions) should then be removed. It is then advised to repeat the visual inspection to ensure that the exclusion algorithm has worked effectively. When using fNIRS in posture and gait, particular care needs to be taken to correct for motion-related artefacts. A large variety of methods are available [105] and can be classified as data-based approaches (e.g. using only fNIRS signals themselves) and approaches correcting for external biomechanical recordings. Among the variety of data-based approaches for removing motion artefacts (Table S1), spline interpolation [106], wavelet-based filters [107-110], or hybrid filter methods [111] are shown 
to be the most promising and powerful methods. To date, there is no consensus on the most effective filter methods to reduce motion artefacts in posture and gait tasks (e.g. low frequency components associated with postural sway, high vertical accelerations associated with foot strikes when walking). This is an important area for future fNIRS research.

\section{Correction of physiological artefacts and superficial layer contamination}

To correct for physiological artefacts, such as heart rate ( 0.5 to $2.0 \mathrm{~Hz}$ ), low-frequency components from blood pressure changes (Mayer waves) $(0.07$ to $0.13 \mathrm{~Hz}$ ) and respiration ( 0.2 to $0.4 \mathrm{~Hz})[73,90,105,112-115]$, a variety of filtering methods have been proposed (Table S1). High-pass and low-pass filters are commonly used to eliminate other sources of noise, but the applied cut-off frequencies should be chosen carefully in order to avoid the removal of stimulus-dependent hemodynamic responses $[61,104,116]$. The cut-off frequency of highpass filters is commonly set at $\sim 0.01 \mathrm{~Hz}$ to remove instrumental-related artefacts and vascular endothelial regulations $[117,118]$ and should be adopted for trials of extended durations (e.g. longer than 100s) [117]. Low-pass filters are commonly used to remove physiological oscillations (e.g., heart rate and/or Mayer waves). A cut-off frequency higher than the stimulus frequency and lower than the frequency of Mayer waves $(<0.1 \mathrm{~Hz})$ is recommended [117]. As alternative to bandpass filters, Savitzky-Golay filters [119] can be used for the purpose of smoothing the data, to increase the precision of the data without distorting the signal tendency. This is achieved, through convolution which can also be used in fNIRS studies [120-122]. Figure 3 provides examples of raw and filtered hemodynamic data.

In addition, the detected fNIRS signals contain both the cerebral hemodynamic activity (of interest) and also extracerebral hemodynamic activity originating from vascularized scalp and 
skull tissue $[90,123,124]$. Sympathetic activity and blood pressure changes associated with posture and gait tasks can result in changes that are not directly task-related. This may require the elimination of the extracerebral hemodynamic activity. Such activity can be filtered to an extent via techniques such as wavelet-based filtering or filters based on principal component analysis [125]. However, a more direct and recently commercially available method involves the application of short-separation channels $(0.5-1 \mathrm{~cm})$ which measure the extracerebral activity alone, so that it may be removed from the total fNIRS signal $[61,126]$. In this regard, it should be noted that the data quality of short-separation channels need to be acceptable, otherwise additional error is introduced [127]. While short-separation channels are a powerful tool to account for systemic physiological artefacts in fNIRS studies, many commercially available systems have fixed optode distances and do not allow for capturing short-separation channels. Approaches to deal with other systemic confounders (e.g., changes in blood pressure or arterial partial pressure of carbon dioxide) have been suggested [128], but have yet to be examined in studies investigating posture or gait [61].

\section{Consideration of the differential path length factor}

The differential path length factor (DPF) is a dimensionless correction factor used in the modified Beer-Lambert law to calculate the concentration of the chromophores (e.g. HbO2 and $\mathrm{HHb})[129,130]$. An inaccurately determined DPF can cause serious cross-talk error [131]. In the modified Beer-Lambert law, the DPF is needed to account for the scatter-dependent increase of optical path length occurring in biological tissue [132-135]. The DPF exhibits large inter-individual heterogeneity [134, 136-138] and is influenced by a variety of factors (see Table S1 for a list). It should be noted that ageing and pathology-related changes in DPF values (e.g. in Parkinson's disease or stroke) are not well-investigated and there is currently, to the 
best of our knowledge, no equation available to account for this. Hence, caution should be paid when comparing findings between groups entailing different pathologies [70]. Recent findings show block design protocols involving highly validated and reliable tasks (e.g. dualtask walking) might be robust to variations in conversion parameters (used in the BeerLambert law, including the DPF) and different low-pass filter applications [139]. Yet, to ensure data repeatability and comparison, it is important to report the parameter values used in conversion to $\mathrm{HbO} 2$ and $\mathrm{HHb}$ such as DPF and molar extinction coefficients.

\section{Outcome measures, validity and reliability}

When using fNIRS, $\mathrm{HbO} 2$ and $\mathrm{HHb}$ outcomes are generally expressed in units of micro-molar concentration. These measures reflect the change in hemoglobin chromophore concentrations (i.e., neural activity) in the measured cortical regions between the task and baseline condition. Some studies have reported only $\mathrm{HbO} 2$ concentration changes as a measure of direct metabolism of the neural tissues. $\mathrm{HbO} 2$ measures are also more expressive of change due to a higher signal-to-noise ratio than $\mathrm{HHb}[140,141] . \mathrm{HbO} 2$, however, has been shown to be more susceptible to systemic contributions (i.e., increased heart rate) that may not be associated with the task performed $[123,142]$. Thus it is recommended to also report changes in $\mathrm{HHb}$ which have been shown to correlate closely with the BOLD signal [143]. Furthermore, there is evidence that the strength of the correlation between $\mathrm{HbO} 2$ and $\mathrm{HHb}$ is a marker of the amount of artefact affecting the signal [144].

By definition, $\mathrm{HbO} 2$ and $\mathrm{HHb}$ exist in equilibrium, such that an increase in one results in a stoichiometric decrease in the other. But this explanation is only valid if regional blood volume is constant. Much of the available research using fNIRS during gait and posture is on older 
adults $[62,63,66,68,69,71]$ and neurological patients $[59,63,66,68,145]$. These populations often have asymmetrical neural pathologies and vascular disease, which may affect hemodynamics. As such, additional measures have been calculated from $\mathrm{HbO} 2$ and $\mathrm{HHb}$. These include for example, the total hemoglobin ( $\mathrm{HbTotal}=\mathrm{HbO} 2+\mathrm{HHb})$, the tissue oxygenation index which may be expressed as the change in $\mathrm{HbO} 2$ relative to the change in $\mathrm{HHb}[146]$, the ratio of $\mathrm{HbO} 2$ to $\mathrm{HbTotal}[53,147]$, the difference between hemoglobin species $(\mathrm{HbDiff}=\mathrm{HbO} 2-\mathrm{HHb})[31]$ and the regional cortical activation ratio ( $\mathrm{HbO} 2$ measured at a single channel over the ROI divided by average $\mathrm{HbO} 2$ of all channels multiplied by 100) [33]. These measures reflect the systems' ability to utilize (consumption) and replenish (supply) $\mathrm{HbO} 2$ and provide additional insight into task activity and performance. Studies have used different outcome measures to quantify fNIRS data: mean values, median values, peak values, area under the curve, slope, time to peak (see in reviews $[70,104]$ ); their choice generally relate to the distribution of the data and the research question. Regardless of the choice of outcome measure, measures of variability such as standard deviation, standard error, confidence interval, range or interquartile range should always be provided.

\section{Validity and Reliability}

Numerous studies have been conducted to cross-validate fNIRS through comparison with other modalities. Several studies have shown comparable fNIRS signals to functional MRI $[148,149]$ when measured simultaneously (see [150] for a review). Brain activations have also been compared between similar tasks, such as imagined balance/gait tasks in an MRI scanner versus actual balance/gait tasks with fNIRS (see [72] for a review), and stepping movements while supine in an MRI scanner versus upright stepping using fNIRS [151]. While similarities were found within these studies, the inherent posture-related difference between the tasks 
(i.e. supine versus upright) resulted in many differences in regional activation, not necessarily reflective of the task assessed but rather of the method of assessment. In order to further validate fNIRS for balance and gait tasks, studies have used other portable devices such as electroencephalography $[152,153]$ for comparison. However, the properties of hemodynamic response versus electrical physiological response again, are quite different. Thus, cross-validation of fNIRS against other instruments during balance and gait remains a challenge which should be further explored.

Sensitivity and specificity are further important validity components of fNIRS measures. Determination of sensitivity and specificity of fNIRS devices leads to information about the credibility of outcomes [154]. This knowledge may allow assessment of hemispheric asymmetry during locomotion tasks that have, as of yet, not been investigated with fNIRS in relation to physical training interventions [22]. Theories about hemisphere behaviour during locomotion; e.g. the complementary hypothesis [155] and the compensation hypothesis [156, 157], could be tested in ecologically valid scenarios provided fNIRS shows acceptable levels of specificity and sensitivity.

Despite the increasing number of published fNIRS studies assessing posture and gait (e.g. [58, 60-72]), only a few papers reported test-retest reliability. Studies exploring this important attribute with motor tasks (i.e., handgrip tasks in people with and without traumatic brain injury [158]; digit manipulation in healthy people [84]) have reported good to moderate testretest reliability of fNIRS data in the prefrontal and motor cortices. These studies have also shown that both task and signal type influence reliability. $\mathrm{HbO} 2$ signals were more reliable overall, than $\mathrm{HHb}$ signals, while tasks involving larger movements were less reliable. These 


\section{Conclusions and future directions}

fNIRS research in gait and posture is in its relative infancy. This consensus statement represents the current state of knowledge and will require updating as new evidence is produced. We provide a set of guidelines for research but by all means do not intend to 
negate novel fNIRS evidence development. Nonetheless, at the time when research in this area is expanding, it is important to ensure standardization and replication thus, transparency is essential. A number of key components are important for replication of fNIRS research. These include detailing the method of data collection, device specification and signal processing techniques (Table S1).

fNIRS relies on an external placement of recording optodes to guide signal interpretation [80, 160]. An accurate description of the relations between external anatomical landmarks on the scalp and the cortical anatomy beneath is therefore crucial to draw valid conclusions from the measured brain activity with fNIRS [161]. Robust functional inference from the recorded signals can also be facilitated by averaging across channels of ROIs and trials $[61,104,160]$. Different methods have been suggested to determine such ROIs [160, 162]. The choice of ROI and location of the optodes can both impact interpretation of the results.

As a result of certain neurological conditions, the interpretation of brain activation across certain ROls may be problematic. Currently, it is unclear if there are abnormal hemodynamic responses over lesioned areas or peri-lesional areas. Some groups have reported abnormalities in neurovascular coupling post-stroke $[163,164]$ and in near infrared lighttissue interaction in the case of hematomas [165]. This may challenge interpretation as suboptimal neurovascular coupling might be a result of the actual brain pathology (e.g. ischemic regions, arteriosclerosis) or pathological brain function (e.g. neural recruitment or compensation). As one example, we can consider how an asymmetrical brain pathology can impact bilateral activities such as balance and gait. It is therefore strongly recommended to provide explicit and informative definitions for ROls including justification of the number and 
location of channels. In addition, for studies including clinical groups, a description of any brain lesions present and their proximity to fNIRS channels should be provided.

All processing steps and any assumptions made (e.g. the DPF value) should be clearly outlined in reports of fNIRS data. Channel-wise analyses may be impacted by variations in head sizes and shapes between participants. This should be taken into consideration. Methods used for channel localization on the scalp, as well as their spatial registration technique should be detailed. To move the field forward, it is essential to find techniques to account for anatomical anomalies to ensure valid findings. Exploration beyond the single ROI is extremely interesting and includes investigating functional connectomes in a similar way to fMRI [166]. This area is still not developed in the field of fNIRS [167] mainly since this type of approach requires multiple optode locations to cover the whole brain. Recently introduced devices offer whole brain fNIRS coverage, as such, we expect this area will grow and complement the existing neuroimaging literature.

fNIRS data collection methods require repeated trials, which over time, can jeopardize signal quality by reducing signal-to-noise ratio and eventually leading to missing data [89]. Moreover, trials severely contaminated by motion artefacts and/or strong physiological noise are commonly rejected, whether automatically or based on visual inspection [168]. An a priori approach to data removal should be set. The amount of missing data (i.e. number of excluded channels, trials, and/or participants) and how this was accounted for in the analysis should be transparent in the reporting of fNIRS studies. Similarly, the software and specific processing pipelines used should also be described in order to ensure reproducibility of fNIRS findings. Future studies that systematically compare different filter methods are necessary before an 
evidence-based recommendation can be given. Models incorporating multiple physiological confounders may help to better identify the physiological origin of signal changes and help to further elucidate neural function [90]. Table 1 provides a summary of key point recommendations and considerations while Table S1 provides more specific guidance regarding methodological details that should be reported in order to enhance interpretation of research findings.

Inter-individual differences in cognitive, psychological and physical functions are highly significant not only across disease populations but also in normal aging. Among healthy older adults, variables such as gender and stress [169], gait abnormalities [170], levels of fatigue [171] as well as structural brain differences in grey matter volume [27] and white matter integrity [172] have major effects on fNIRS-derived hemodynamic responses. Moreover, improved efficiency in fNIRS-derived activation patterns due to practice in one session [26] was greatly affected by the presence of fear of falls [173]. Hence, due to the inherent heterogeneity in disease populations and healthy older adults the sample size should be carefully considered and resources should be explicitly allocated to maximize the number of participants. Furthermore, detailed characterization of the participants in terms of relevant demographic and clinical variables should be provided. Such information will be critical for replication and test-retest reliability studies as well as for investigations that are specifically designed to evaluate the utility of fNIRS as primary or secondary outcome measure in clinical trials.

Lastly, to advance the field, researchers should consider data sharing through open science repositories. This will allow researchers to compare their data and processing algorithms with 
others directly, instead of indirectly through published reports. Such repositories are becoming increasingly common in the imaging field such as in MRI research (e.g., International Data-sharing Neuroimaging Initiative: INDI from the Consortium for Reliability and Reproducibility (CoRR) [174] and the CBS Neuroimaging Repository [175]) as they can stimulate the development of data processing tools, facilitate reproducibility and collaboration. The added advantage of open science repositories is that it makes research products open to everyone. This in turn accelerates the identification and understanding of the neural underpinnings involved during posture and gait tasks. 
Author contributions: JM and $\mathrm{AM}$ designed the concept of this manuscript, led the collaborative writing and reviewing efforts, and edited the final draft of the manuscript. All authors contributed to the redaction and reviewing of the manuscript. 


\section{REFERENCES}

1. H. Owen-Reece, M. Smith, C.E. Elwell, J.C. Goldstone, Near infrared spectroscopy, Br J Anaesth. 82 (1999) 418-426. 10.1093/bja/82.3.418.

2. T. Csipo, P. Mukli, A. Lipecz, S. Tarantini, D. Bahadli, O. Abdulhussein, et al., Assessment of age-related decline of neurovascular coupling responses by functional near-infrared spectroscopy (fNIRS) in humans, Geroscience. 41 (2019) 495-509. 10.1007/s11357-01900122-x.

3. M. Fabiani, B.A. Gordon, E.L. Maclin, M.A. Pearson, C.R. Brumback-Peltz, K.A. Low, et al., Neurovascular coupling in normal aging: a combined optical, ERP and fMRI study, Neuroimage. 85 Pt 1 (2014) 592-607. 10.1016/j.neuroimage.2013.04.113.

4. J. Steinbrink, A. Villringer, F. Kempf, D. Haux, S. Boden, H. Obrig, Illuminating the BOLD signal: combined fMRI-fNIRS studies, Magn Reson Imaging. 24 (2006) 495-505. 10.1016/j.mri.2005.12.034.

5. H. Karim, B. Schmidt, D. Dart, N. Beluk, T. Huppert, Functional near-infrared spectroscopy (fNIRS) of brain function during active balancing using a video game system, Gait Posture. 35 (2012) 367-372. http://dx.doi.org/10.1016/j.gaitpost.2011.10.007.

6. T. Huppert, B. Schmidt, N. Beluk, J. Furman, P. Sparto, Measurement of brain activation during an upright stepping reaction task using functional near-infrared spectroscopy, Hum Brain Mapp. 34 (2013) 2817-2828. http://dx.doi.org/10.1002/hbm.22106.

7. A.L. Rosso, M. Cenciarini, P.J. Sparto, P.J. Loughlin, J.M. Furman, T.J. Huppert, Neuroimaging of an attention demanding dual-task during dynamic postural control, Gait Posture. 57 (2017) 193-198. http://dx.doi.org/10.1016/j.gaitpost.2017.06.013.

8. S. Basso Moro, S. Bisconti, M. Muthalib, M. Spezialetti, S. Cutini, M. Ferrari, et al., A semiimmersive virtual reality incremental swing balance task activates prefrontal cortex: A functional near-infrared spectroscopy study, Neurolmage. 85 (2014) 451-460. http://dx.doi.org/10.1016/j.neuroimage.2013.05.031. 
9. A.B. Rosen, J.M. Yentes, M.L. McGrath, A.C. Maerlender, S.A. Myers, M. Mukherjee, Alterations in Cortical Activation Among Individuals With Chronic Ankle Instability During Single-Limb Postural Control, J Athl Train. 54 (2019) 718-726. http://dx.doi.org/10.4085/10626050-448-17.

10. W.P. Teo, A.M. Goodwill, A.M. Hendy, M. Muthalib, H. Macpherson, Sensory manipulation results in increased dorsolateral prefrontal cortex activation during static postural balance in sedentary older adults: An fNIRS study, Brain Behav. 8 (2018) http://dx.doi.org/10.1002/brb3.1109.

11. A.C. de Lima-Pardini, G.A. Zimeo Morais, J.B. Balardin, D.B. Coelho, N.M. Azzi, L.A. Teixeira, et al., Measuring cortical motor hemodynamics during assisted stepping - An fNIRS feasibility study of using a walker, Gait Posture. $56 \quad$ (2017) 112-118. http://dx.doi.org/10.1016/j.gaitpost.2017.05.018.

12. A. Mirelman, I. Maidan, H. Bernad-Elazari, F. Nieuwhof, M. Reelick, N. Giladi, et al., Increased frontal brain activation during walking while dual tasking: An fNIRS study in healthy young adults, J Neuroeng Rehabil. 11 (2014) http://dx.doi.org/10.1186/1743-0003-11-85.

13. R. Holtzer, J.R. Mahoney, M. Izzetoglu, C. Wang, S. England, J. Verghese, Online fronto-cortical control of simple and attention-demanding locomotion in humans, Neurolmage. 112 (2015) 152-159. http://dx.doi.org/10.1016/j.neuroimage.2015.03.002.

14. M. Chen, S. Pillemer, S. England, M. Izzetoglu, J.R. Mahoney, R. Holtzer, Neural correlates of obstacle negotiation in older adults: An fNIRS study, Gait Posture. 58 (2017) 130-135. http://dx.doi.org/10.1016/j.gaitpost.2017.07.043.

15. A. Mirelman, I. Maidan, H. Bernad-Elazari, S. Shustack, N. Giladi, J.M. Hausdorff, Effects of aging on prefrontal brain activation during challenging walking conditions, Brain Cogn. 115 (2017) 41-46. http://dx.doi.org/10.1016/j.bandc.2017.04.002. 
16. I. Maidan, S. Shustak, T. Sharon, H. Bernad-Elazari, N. Geffen, N. Giladi, et al., Prefrontal cortex activation during obstacle negotiation: What's the effect size and timing?, Brain Cogn. 122 (2018) 45-51. http://dx.doi.org/10.1016/j.bandc.2018.02.006.

17. K.A. Hawkins, E.J. Fox, J.J. Daly, D.K. Rose, E.A. Christou, T.E. McGuirk, et al., Prefrontal overactivation during walking in people with mobility deficits: Interpretation and functional implications, Hum Mov Sci. 59 (2018) 46-55. http://dx.doi.org/10.1016/j.humov.2018.03.010.

18. M.J. Kurz, T.W. Wilson, D.J. Arpin, Stride-time variability and sensorimotor cortical activation $\begin{array}{llll}\text { during } \quad \text { walking, } & \text { Neurolmage. } & 59 & \text { (2012) }\end{array}$ http://dx.doi.org/10.1016/j.neuroimage.2011.08.084.

19. R. Beurskens, I. Helmich, R. Rein, O. Bock, Age-related changes in prefrontal activity during walking in dual-task situations: A fNIRS study, Int J Psychophysiol. 92 (2014) 122-128. http://dx.doi.org/10.1016/j.ijpsycho.2014.03.005.

20. K.L.M. Koenraadt, E.G.J. Roelofsen, J. Duysens, N.L.W. Keijsers, Cortical control of normal gait and precision stepping: An fNIRS study, Neurolmage. 85 (2014) 415-422. http://dx.doi.org/10.1016/j.neuroimage.2013.04.070.

21. D. Meester, E. Al-Yahya, H. Dawes, P. Martin-Fagg, C. Pinon, Associations between prefrontal cortex activation and H-reflex modulation during dual task gait, Front Hum Neurosci. 8 (2014) http://dx.doi.org/10.3389/fnhum.2014.00078.

22. P. Eggenberger, M. Wolf, M. Schumann, E.D. de Bruin, Exergame and balance training modulate prefrontal brain activity during walking and enhance executive function in older adults, Front Aging Neurosci. 8 (2016) http://dx.doi.org/10.3389/fnagi.2016.00066.

23. S.A. Fraser, O. Dupuy, P. Pouliot, F. Lesage, L. Bherer, Comparable cerebral oxygenation patterns in younger and older adults during dual-task walking with increasing load, Front Aging Neurosci. 8 (2016) http://dx.doi.org/10.3389/fnagi.2016.00240. 
24. T. Harada, I. Miyai, M. Suzuki, K. Kubota, Gait capacity affects cortical activation patterns related to speed control in the elderly, Exp Brain Res. 193 (2009) 445-454. http://dx.doi.org/10.1007/s00221-008-1643-y.

25. C.J. George, J. Verghese, M. Izzetoglu, C. Wang, R. Holtzer, The effect of polypharmacy on prefrontal cortex activation during single and dual task walking in community dwelling older adults, Pharmacol Res. 139 (2019) 113-119. http://dx.doi.org/10.1016/j.phrs.2018.11.007.

26. R. Holtzer, M. Izzetoglu, M. Chen, C. Wang, Distinct fNIRS-Derived HbO2 Trajectories During the Course and Over Repeated Walking Trials Under Single- and Dual-Task Conditions: Implications for Within Session Learning and Prefrontal Cortex Efficiency in Older Adults, J Gerontol A Biol Sci Med Sci. 74 (2019) 1076-1083. http://dx.doi.org/10.1093/gerona/gly181.

27. M.E. Wagshul, M. Lucas, K. Ye, M. Izzetoglu, R. Holtzer, Multi-modal neuroimaging of dualtask walking: Structural MRI and fNIRS analysis reveals prefrontal grey matter volume moderation of brain activation in older adults, Neurolmage. 189 (2019) 745-754. http://dx.doi.org/10.1016/j.neuroimage.2019.01.045.

28. S. Stuart, L. Alcock, L. Rochester, R. Vitorio, A. Pantall, Monitoring multiple cortical regions during walking in young and older adults: Dual-task response and comparison challenges, Int. J. Psychophysiol. 135 (2019) 63-72. http://dx.doi.org/10.1016/j.ijpsycho.2018.11.006.

29. F.G. Metzger, A.C. Ehlis, F.B. Haeussinger, P. Schneeweiss, J. Hudak, A.J. Fallgatter, et al., Functional brain imaging of walking while talking - An fNIRS study, Neuroscience. 343 (2017) 85-93. http://dx.doi.org/10.1016/j.neuroscience.2016.11.032.

30. R. Holtzer, J.R. Mahoney, M. Izzetoglu, K. Izzetoglu, B. Onaral, J. Verghese, fNIRS study of walking and walking while talking in young and old individuals, J Gerontol A Biol Sci Med Sci. 66 (2011) 879-887. 10.1093/gerona/g|r068.

31. C.F. Lu, Y.C. Liu, Y.R. Yang, Y.T. Wu, R.Y. Wang, Maintaining gait performance by cortical activation during dual-task interference: A functional near-infrared spectroscopy study, PLoS One. 10 (2015) http://dx.doi.org/10.1371/journal.pone.0129390. 
32. M. Suzuki, I. Miyai, T. Ono, K. Kubota, Activities in the frontal cortex and gait performance are modulated by preparation. An fNIRS study, Neurolmage. 39 (2008) 600-607. http://dx.doi.org/10.1016/j.neuroimage.2007.08.044.

33. M. Suzuki, I. Miyai, T. Ono, I. Oda, I. Konishi, T. Kochiyama, et al., Prefrontal and premotor cortices are involved in adapting walking and running speed on the treadmill: an optical imaging study, Neuroimage. 23 (2004) 1020-1026. 10.1016/j.neuroimage.2004.07.002.

34. V. Belluscio, S. Stuart, E. Bergamini, G. Vannozzi, M. Mancini, The Association between Prefrontal Cortex Activity and Turning Behavior in People with and without Freezing of Gait, Neuroscience. 416 (2019) 168-176. http://dx.doi.org/10.1016/j.neuroscience.2019.07.024.

35. I. Maidan, H. Bernad-Elazari, E. Gazit, N. Giladi, J.M. Hausdorff, A. Mirelman, Changes in oxygenated hemoglobin link freezing of gait to frontal activation in patients with Parkinson disease: an fNIRS study of transient motor-cognitive failures, J Neurol. 31 (2015) http://dx.doi.org/10.1007/s00415-015-7650-6.

36. I. Maidan, H. Bernad-Elazari, N. Giladi, J.M. Hausdorff, A. Mirelman, When is Higher Level Cognitive Control Needed for Locomotor Tasks Among Patients with Parkinson's Disease?, Brain Topogr. 30 (2017) 531-538. http://dx.doi.org/10.1007/s10548-017-0564-0.

37. I. Maidan, F. Nieuwhof, H. Bernad-Elazari, B.R. Bloem, N. Giladi, J.M. Hausdorff, et al., Evidence for Differential Effects of 2 Forms of Exercise on Prefrontal Plasticity During Walking in Parkinson's Disease, Neurorehabil Neural Repair. 32 (2018) 200-208. http://dx.doi.org/10.1177/1545968318763750.

38. I. Maidan, F. Nieuwhof, H. Bernad-Elazari, M.F. Reelick, B.R. Bloem, N. Giladi, et al., The Role of the Frontal Lobe in Complex Walking among Patients with Parkinson's Disease and Healthy Older Adults: An fNIRS Study, Neurorehabil Neural Repair. 30 (2016) 963-971. http://dx.doi.org/10.1177/1545968316650426. 
39. S. Stuart, V. Belluscio, J.F. Quinn, M. Mancini, Pre-frontal cortical activity during walking and turning is reliable and differentiates across young, older adults and people with Parkinson's disease, Front Neurol. 10 (2019) http://dx.doi.org/10.3389/fneur.2019.00536.

40. S. Stuart, M. Mancini, Prefrontal Cortical Activation With Open and Closed-Loop Tactile Cueing When Walking and Turning in Parkinson Disease: A Pilot Study, J Neurolc Phys Ther. 14 (2019) http://dx.doi.org/10.1097/NPT.0000000000000286.

41. P.C. Thumm, I. Maidan, M. Brozgol, S. Shustak, E. Gazit, S. Shema Shiratzki, et al., Treadmill walking reduces pre-frontal activation in patients with Parkinson's disease, Gait Posture. 62 (2018) 384-387. http://dx.doi.org/10.1016/j.gaitpost.2018.03.041.

42. S.A. Chatterjee, E.J. Fox, J.J. Daly, D.K. Rose, S.S. Wu, E.A. Christou, et al., Interpreting prefrontal recruitment during walking after stroke: Influence of individual differences in mobility and cognitive function, Front Hum Neurosci. 13 (2019) http://dx.doi.org/10.3389/fnhum.2019.00194.

43. H. Fujimoto, M. Mihara, N. Hattori, M. Hatakenaka, T. Kawano, H. Yagura, et al., Cortical changes underlying balance recovery in patients with hemiplegic stroke, Neurolmage. 85 (2014) 547-554. http://dx.doi.org/10.1016/j.neuroimage.2013.05.014.

44. E. Hermand, B. Tapie, O. Dupuy, S. Fraser, M. Compagnat, J.Y. Salle, et al., Prefrontal cortex activation during dual task with increasing cognitive load in subacute stroke patients: A pilot study, Front Aging Neurosci. 10 (2019) http://dx.doi.org/10.3389/fnagi.2019.00160.

45. Y.C. Liu, Y.R. Yang, Y.A. Tsai, R.Y. Wang, C.F. Lu, Brain Activation and Gait Alteration during Cognitive and Motor Dual Task Walking in Stroke-A Functional Near-Infrared Spectroscopy Study, IEEE Trans Neural Syst Rehabil Eng. $26 \quad$ (2018) 2416-2423. http://dx.doi.org/10.1109/TNSRE.2018.2878045.

46. M. Mihara, I. Miyai, M. Hatakenaka, K. Kubota, S. Sakoda, Sustained prefrontal activation during ataxic gait: A compensatory mechanism for ataxic stroke?, Neurolmage. 37 (2007) 1338-1345. http://dx.doi.org/10.1016/j.neuroimage.2007.06.014. 
47. M. Mihara, I. Miyai, N. Hattori, M. Hatakenaka, H. Yagura, et al., Cortical control of postural balance in patients with hemiplegic stroke, NeuroReport. 18 (2012) http://dx.doi.org/10.1097/WNR.0b013e328351757b.

48. M. Rea, M. Rana, N. Lugato, P. Terekhin, L. Gizzi, D. Brotz, et al., Lower limb movement preparation in chronic stroke: A pilot study toward an fNIRS-BCl for gait rehabilitation, $\begin{array}{lllll}\text { Neurorehabil } & \text { Neural } & \text { Repair. } & 28 & \text { (2014) }\end{array}$ http://dx.doi.org/10.1177/1545968313520410.

49. G. Chaparro, J.M. Balto, B.M. Sandroff, R. Holtzer, M. Izzetoglu, R.W. Motl, et al., Frontal brain activation changes due to dual-tasking under partial body weight support conditions in older adults with multiple sclerosis, J Neuroeng Rehabil. 14 (2017) http://dx.doi.org/10.1186/s12984-017-0280-8.

50. M.E. Hernandez, R. Holtzer, G. Chaparro, K. Jean, J.M. Balto, B.M. Sandroff, et al., Brain activation changes during locomotion in middle-aged to older adults with multiple sclerosis, J Neurol Sci. 370 (2016) 277-283. http://dx.doi.org/10.1016/j.jns.2016.10.002.

51. M.E. Hernandez, E. O'Donnell, G. Chaparro, R. Holtzer, M. Izzetoglu, B.M. Sandroff, et al., Brain activation changes during balance- And attention-demanding tasks in middle- And older-aged adults with multiple sclerosis, Motor Control. 23 (2019) 498-517. http://dx.doi.org/10.1123/mc.2018-0044.

52. S. Saleh, B.M. Sandroff, T. Vitiello, O. Owoeye, A. Hoxha, P. Hake, et al., The role of premotor areas in dual tasking in healthy controls and persons with multiple sclerosis: An fNIRS imaging study, Front Behav Neurosci. 12 (2018) http://dx.doi.org/10.3389/fnbeh.2018.00296.

53. D.J. Clark, E.A. Christou, S.A. Ring, J.B. Williamson, L. Doty, Enhanced somatosensory feedback reduces prefrontal cortical activity during walking in older adults, J Gerontol A Biol Sci Med Sci. 69 (2014) 1422-1428. http://dx.doi.org/10.1093/gerona/glu125. 
54. R. Holtzer, B.C. Rakitin, J. Steffener, J. Flynn, A. Kumar, Y. Stern, Age effects on load-dependent brain activations in working memory for novel material, Brain Res. 1249 (2009) 148-161. 10.1016/j.brainres.2008.10.009.

55. Y. Stern, What is cognitive reserve? Theory and research application of the reserve concept, J Int Neuropsychol Soc. 8 (2002) 448-460.

56. Y. Stern, Cognitive reserve, Neuropsychologia. 47 (2009) 2015-2028. 10.1016/j.neuropsychologia.2009.03.004.

57. S.M. Daselaar, V. Iyengar, S.W. Davis, K. Eklund, S.M. Hayes, R.E. Cabeza, Less wiring, more firing: low-performing older adults compensate for impaired white matter with greater neural activity, Cereb Cortex. 25 (2015) 983-990. 10.1093/cercor/bht289.

58. A. Berger, F. Horst, S. Muller, F. Steinberg, M. Doppelmayr, Current state and future prospects of EEG and fNIRS in robot-assisted gait rehabilitation: A brief review, Front Hum Neurosci. 13 (2019) http://dx.doi.org/10.3389/fnhum.2019.00172.

59. V. Gramigna, G. Pellegrino, A. Cerasa, S. Cutini, R. Vasta, G. Olivadese, et al., Near-Infrared Spectroscopy in Gait Disorders: Is It Time to Begin?, Neurorehabil Neural Repair. 31 (2017) 402-412. http://dx.doi.org/10.1177/1545968317693304.

60. F. Herold, P. Wiegel, D. Hamacher, L. Schega, Brain activity during walking: A systematic $\begin{array}{lllll}\text { review, Neurosci } & \text { Biobehav } & \text { 310-327. }\end{array}$ http://dx.doi.org/10.1016/j.neubiorev.2015.08.002.

61. F. Herold, P. Wiegel, F. Scholkmann, A. Thiers, D. Hamacher, L. Schega, Functional nearinfrared spectroscopy in movement science: A systematic review on cortical activity in $\begin{array}{lllll}\text { postural and walking tasks, Neurophotonics. } & 4 & \text { (2017) }\end{array}$ http://dx.doi.org/10.1117/1.NPh.4.4.041403.

62. R. Holtzer, N. Epstein, J.R. Mahoney, M. Izzetoglu, H.M. Blumen, Neuroimaging of mobility in aging: a targeted review, J Gerontol A Biol Sci Med Sci. 69 (2014) 1375-1388. http://dx.doi.org/10.1093/gerona/glu052. 
63. M. Kahya, S. Moon, M. Ranchet, R.R. Vukas, K.E. Lyons, R. Pahwa, et al., Brain activity during dual task gait and balance in aging and age-related neurodegenerative conditions: A systematic review, Exp Gerontol. 128 (2019) 110756. 10.1016/j.exger.2019.110756.

64. D.R. Leff, F. Orihuela-Espina, C.E. Elwell, T. Athanasiou, D.T. Delpy, A.W. Darzi, et al., Assessment of the cerebral cortex during motor task behaviours in adults: A systematic review of functional near infrared spectroscopy (fNIRS) studies, Neurolmage. 54 (2011) 2922-2936. http://dx.doi.org/10.1016/j.neuroimage.2010.10.058.

65. M. Mihara, I. Miyai, Review of functional near-infrared spectroscopy in neurorehabilitation, Neurophotonics. 3 (2016) http://dx.doi.org/10.1117/1.NPh.3.3.031414.

66. P.H.S. Pelicioni, M. Tijsma, S.R. Lord, J. Menant, Prefrontal cortical activation measured by fNIRS during walking: effects of age, disease and secondary task, PeerJ. 7 (2019) e6833. 10.7717/peerj.6833.

67. V. Quaresima, M. Ferrari, A Mini-Review on Functional Near-Infrared Spectroscopy (fNIRS): Where Do We Stand, and Where Should We Go?, Photonics. 6 (2019)

68. S. Stuart, R. Vitorio, R. Morris, D.N. Martini, P.C. Fino, M. Mancini, Cortical activity during walking and balance tasks in older adults and in people with Parkinson's disease: A structured review, Maturitas. 113 (2018) 53-72. http://dx.doi.org/10.1016/j.maturitas.2018.04.011.

69. C. Udina, S. Avtzi, T. Durduran, R. Holtzer, A.L. Rosso, C. Castellano-Tejedor, et al., Functional Near-Infrared Spectroscopy to Study Cerebral Hemodynamics in Older Adults During Cognitive and Motor Tasks: A Review, Front Aging Neurosci. 11 (2019) 367. 10.3389/fnagi.2019.00367.

70. R. Vitorio, S. Stuart, L. Rochester, L. Alcock, A. Pantall, fNIRS response during walking - Artefact or cortical activity? A systematic review, Neurosci Biobehav Rev. 83 (2017) 160-172. http://dx.doi.org/10.1016/j.neubiorev.2017.10.002.

71. J. Wilson, L. Allcock, R. Mc Ardle, J.P. Taylor, L. Rochester, The neural correlates of discrete gait characteristics in ageing: A structured review, Neurosci Biobehav Rev. 100 (2019) 344369. http://dx.doi.org/10.1016/j.neubiorev.2018.12.017. 
72. D. Hamacher, F. Herold, P. Wiegel, D. Hamacher, L. Schega, Brain activity during walking: A systematic review, Neurosci Biobehav Rev. $57 \quad$ (2015) 310-327. 10.1016/j.neubiorev.2015.08.002.

73. F. Scholkmann, S. Kleiser, A.J. Metz, R. Zimmermann, J. Mata Pavia, U. Wolf, et al., A review on continuous wave functional near-infrared spectroscopy and imaging instrumentation and methodology, Neuroimage. 85 Pt 1 (2014a) 6-27. 10.1016/j.neuroimage.2013.05.004.

74. L. Wang, H. Ayaz, M. Izzetoglu, B. Onaral, Evaluation of light detector surface area for functional Near Infrared Spectroscopy, Comput Biol Med. 89 (2017) 68-75. 10.1016/j.compbiomed.2017.07.019.

75. X. Cui, S. Bray, A.L. Reiss, Speeded near infrared spectroscopy (NIRS) response detection, PLoS One. 5 (2010) e15474. 10.1371/journal.pone.0015474.

76. M.L. Schroeter, S. Zysset, D.Y. von Cramon, Shortening intertrial intervals in event-related cognitive studies with near-infrared spectroscopy, Neuroimage. 22 (2004) 341-346. 10.1016/j.neuroimage.2003.12.041.

77. I. Tachtsidis, C.E. Elwell, T.S. Leung, C.W. Lee, M. Smith, D.T. Delpy, Investigation of cerebral haemodynamics by near-infrared spectroscopy in young healthy volunteers reveals posturedependent spontaneous oscillations, Physiol Meas. 25 (2004) 437-445. 10.1088/0967$3334 / 25 / 2 / 003$.

78. G.H. Klem, H.O. Luders, H.H. Jasper, C. Elger, The ten-twenty electrode system of the International Federation. The International Federation of Clinical Neurophysiology, Electroencephalogr Clin Neurophysiol Suppl. 52 (1999) 3-6.

79. V. Jurcak, D. Tsuzuki, I. Dan, $10 / 20,10 / 10$, and $10 / 5$ systems revisited: their validity as relative head-surface-based positioning systems, Neuroimage. $34 \quad$ (2007) 1600-1611. 10.1016/j.neuroimage.2006.09.024. 
80. G.A. Zimeo Morais, J.B. Balardin, J.R. Sato, fNIRS Optodes' Location Decider (fOLD): a toolbox for probe arrangement guided by brain regions-of-interest, Sci Rep. 8 (2018) 3341. 10.1038/s41598-018-21716-z.

81. A.K. Singh, M. Okamoto, H. Dan, V. Jurcak, I. Dan, Spatial registration of multichannel multisubject fNIRS data to MNI space without MRI, Neuroimage. 27 (2005) 842-851. 10.1016/j.neuroimage.2005.05.019.

82. A. Alexander-Bloch, J.N. Giedd, E. Bullmore, Imaging structural co-variance between human brain regions, Nat Rev Neurosci. 14 (2013) 322-336. 10.1038/nrn3465.

83. J. Ashburner, J.G. Csernansky, C. Davatzikos, N.C. Fox, G.B. Frisoni, P.M. Thompson, Computerassisted imaging to assess brain structure in healthy and diseased brains, Lancet Neurol. 2 (2003) 79-88. 10.1016/s1474-4422(03)00304-1.

84. S. Dravida, J.A. Noah, X. Zhang, J. Hirsch, Comparison of oxyhemoglobin and deoxyhemoglobin signal reliability with and without global mean removal for digit manipulation motor tasks, Neurophotonics. 5 (2018) 011006. 10.1117/1.NPh.5.1.011006.

85. J.B. Balardin, G.A. Zimeo Morais, R.A. Furucho, L.R. Trambaiolli, J.R. Sato, Impact of communicative head movements on the quality of functional near-infrared spectroscopy signals: negligible effects for affirmative and negative gestures and consistent artifacts related to raising eyebrows, J Biomed Opt. 22 (2017) 46010. 10.1117/1.Jbo.22.4.046010.

86. G.A. Zimeo Morais, F. Scholkmann, J.B. Balardin, R.A. Furucho, R.C.V. de Paula, C.E. Biazoli, Jr., et al., Non-neuronal evoked and spontaneous hemodynamic changes in the anterior temporal region of the human head may lead to misinterpretations of functional near-infrared spectroscopy signals, Neurophotonics. 5 (2018) 011002. 10.1117/1.NPh.5.1.011002.

87. P. Pinti, C. Aichelburg, S. Gilbert, A. Hamilton, J. Hirsch, P. Burgess, et al., A Review on the Use of Wearable Functional Near-Infrared Spectroscopy in Naturalistic Environments, Jpn Psychol Res. 60 (2018) 347-373. 10.1111/jpr.12206. 
88. L. Pollonini, H. Bortfeld, J.S. Oghalai, PHOEBE: a method for real time mapping of optodesscalp coupling in functional near-infrared spectroscopy, Biomed Opt Express. 7 (2016) 51045119. 10.1364/boe.7.005104.

89. F. Orihuela-Espina, D.R. Leff, D.R. James, A.W. Darzi, G.Z. Yang, Quality control and assurance in functional near infrared spectroscopy (fNIRS) experimentation, Phys Med Biol. 55 (2010) 3701-3724. 10.1088/0031-9155/55/13/009.

90. I. Tachtsidis, F. Scholkmann, False positives and false negatives in functional near-infrared spectroscopy: issues, challenges, and the way forward, Neurophotonics. 3 (2016) 030401. 10.1117/1.NPh.3.3.030401.

91. D. Tomasi, E.C. Caparelli, L. Chang, T. Ernst, fMRI-acoustic noise alters brain activation during working memory tasks, Neuroimage. 27 (2005) 377-386. 10.1016/j.neuroimage.2005.04.010.

92. J.M. Baker, D. Rojas-Valverde, R. Gutierrez, M. Winkler, S. Fuhrimann, B. Eskenazi, et al., Portable Functional Neuroimaging as an Environmental Epidemiology Tool: A How-To Guide for the Use of fNIRS in Field Studies, Environ Health Perspect. 125 (2017) 094502. 10.1289/ehp2049.

93. A.J. Metz, S.D. Klein, F. Scholkmann, U. Wolf, Continuous coloured light altered human brain haemodynamics and oxygenation assessed by systemic physiology augmented functional near-infrared spectroscopy, Sci Rep. 7 (2017) 10027. 10.1038/s41598-017-09970-z.

94. F. Scholkmann, T. Hafner, A.J. Metz, M. Wolf, U. Wolf, Effect of short-term colored-light exposure on cerebral hemodynamics and oxygenation, and systemic physiological activity, Neurophotonics. 4 (2017) 045005. 10.1117/1.NPh.4.4.045005.

95. K. Uludag, J. Steinbrink, A. Villringer, H. Obrig, Separability and cross talk: optimizing dual wavelength combinations for near-infrared spectroscopy of the adult head, Neuroimage. 22 (2004) 583-589. 10.1016/j.neuroimage.2004.02.023. 
96. R.K. Almajidy, K. Mankodiya, M. Abtahi, U.G. Hofmann, A Newcomer's Guide to Functional Near Infrared Spectroscopy Experiments, IEEE Rev Biomed Eng. 13 (2020) 292-308. 10.1109/rbme.2019.2944351.

97. M. Schecklmann, A. Mann, B. Langguth, A.C. Ehlis, A.J. Fallgatter, F.B. Haeussinger, The Temporal Muscle of the Head Can Cause Artifacts in Optical Imaging Studies with Functional Near-Infrared Spectroscopy, Front Hum Neurosci. 11 (2017) 456. 10.3389/fnhum.2017.00456.

98. F. Scholkmann, A.J. Metz, M. Wolf, Measuring tissue hemodynamics and oxygenation by continuous-wave functional near-infrared spectroscopy--how robust are the different calculation methods against movement artifacts?, Physiol Meas. 35 (2014b) 717-734. 10.1088/0967-3334/35/4/717.

99. X. Cui, J.M. Baker, N. Liu, A.L. Reiss, Sensitivity of fNIRS measurement to head motion: an applied use of smartphones in the lab, J Neurosci Methods. 245 (2015) 37-43. 10.1016/j.jneumeth.2015.02.006.

100. A. Metz, M. Wolf, P. Achermann, F. Scholkmann, A New Approach for Automatic Removal of Movement Artifacts in Near-Infrared Spectroscopy Time Series by Means of Acceleration Data, Algorithms. 8 (2015) 1052-1075. doi: 10.3390/a8041052.

101. J. Virtanen, T. Noponen, K. Kotilahti, J. Virtanen, R.J. Ilmoniemi, Accelerometer-based method for correcting signal baseline changes caused by motion artifacts in medical near-infrared spectroscopy, J Biomed Opt. 16 (2011) 087005. 10.1117/1.3606576.

102. L. Gagnon, R.J. Cooper, M.A. Yucel, K.L. Perdue, D.N. Greve, D.A. Boas, Short separation channel location impacts the performance of short channel regression in NIRS, Neuroimage. 59 (2012) 2518-2528. 10.1016/j.neuroimage.2011.08.095.

103. T. Sato, I. Nambu, K. Takeda, T. Aihara, O. Yamashita, Y. Isogaya, et al., Reduction of global interference of scalp-hemodynamics in functional near-infrared spectroscopy using short distance probes, Neuroimage. 141 (2016) 120-132. 10.1016/j.neuroimage.2016.06.054. 
104. F. Herold, P. Wiegel, F. Scholkmann, N.G. Muller, Applications of Functional Near-Infrared Spectroscopy (fNIRS) Neuroimaging in Exercise - Cognition Science: A Systematic, Methodology-Focused Review, J Clin Med. 7 (2018) 10.3390/jcm7120466.

105. A. Janani, M. Sasikala, Investigation of different approaches for noise reduction in functional near-infrared spectroscopy signals for brain-computer interface applications., Neural Comput \& Applic. 4 (2017) 10.1007/s00521-017-2961-4.

106. R.J. Cooper, J. Selb, L. Gagnon, D. Phillip, H.W. Schytz, H.K. Iversen, et al., A systematic comparison of motion artifact correction techniques for functional near-infrared spectroscopy, Front Neurosci. 6 (2012) 147. 10.3389/fnins.2012.00147.

107. H.F. Behrendt, C. Firk, C.A. Nelson, 3rd, K.L. Perdue, Motion correction for infant functional near-infrared spectroscopy with an application to live interaction data, Neurophotonics. 5 (2018) 015004. 10.1117/1.NPh.5.1.015004.

108. S. Brigadoi, L. Ceccherini, S. Cutini, F. Scarpa, P. Scatturin, J. Selb, et al., Motion artifacts in functional near-infrared spectroscopy: a comparison of motion correction techniques applied to real cognitive data, Neuroimage. 85 Pt 1 (2014) 181-191. 10.1016/j.neuroimage.2013.04.082.

109. R. Di Lorenzo, L. Pirazzoli, A. Blasi, C. Bulgarelli, Y. Hakuno, Y. Minagawa, et al., Recommendations for motion correction of infant fNIRS data applicable to multiple data sets and acquisition systems, Neuroimage. $200 \quad$ (2019) 511-527. 10.1016/j.neuroimage.2019.06.056.

110. C. Piazza, A. Bacchetta, A. Crippa, M. Mauri, S. Grazioli, G. Reni, et al. Preprocessing Pipeline for fNIRS Data in Children, in: J. Henriques, N. Neves, P. de Carvalho, (Eds), XV Mediterranean Conference on Medical and Biological Engineering and Computing - MEDICON 2019. MEDICON 2019. IFMBE Proceedings, Springer, Cham, 2020, vol 76.

111. S. Jahani, S.K. Setarehdan, D.A. Boas, M.A. Yucel, Motion artifact detection and correction in functional near-infrared spectroscopy: a new hybrid method based on spline interpolation 
method and Savitzky-Golay filtering, Neurophotonics. $5 \quad$ (2018) 015003. 10.1117/1.NPh.5.1.015003.

112. A. Chaddad, Brain Function Diagnosis Enhanced Using Denoised fNIRS Raw Signals, JBiSE. 7 (2014) 218-227. 10.4236/jbise.2014.74025.

113. M.A. Kamran, M.M. Mannan, M.Y. Jeong, Cortical Signal Analysis and Advances in Functional Near-Infrared Spectroscopy Signal: A Review, Front Hum Neurosci. 10 (2016) 261. 10.3389/fnhum.2016.00261.

114. E. Kirilina, N. Yu, A. Jelzow, H. Wabnitz, A.M. Jacobs, I. Tachtsidis, Identifying and quantifying main components of physiological noise in functional near infrared spectroscopy on the prefrontal cortex, Front Hum Neurosci. 7 (2013) 864. 10.3389/fnhum.2013.00864.

115. F. Scholkmann, S. Spichtig, T. Muehlemann, M. Wolf, How to detect and reduce movement artifacts in near-infrared imaging using moving standard deviation and spline interpolation, Physiol Meas. 31 (2010) 649-662. 10.1088/0967-3334/31/5/004.

116. T.J. Huppert, S.G. Diamond, M.A. Franceschini, D.A. Boas, HomER: a review of time-series analysis methods for near-infrared spectroscopy of the brain, Appl Opt. 48 (2009) D280-298. $10.1364 /$ ao. $48.00 \mathrm{~d} 280$.

117. P. Pinti, F. Scholkmann, A. Hamilton, P. Burgess, I. Tachtsidis, Current Status and Issues Regarding Pre-processing of fNIRS Neuroimaging Data: An Investigation of Diverse Signal Filtering Methods Within a General Linear Model Framework, Front Hum Neurosci. 12 (2018) 505. 10.3389/fnhum.2018.00505.

118. M.A. Yucel, J. Selb, C.M. Aasted, P.Y. Lin, D. Borsook, L. Becerra, et al., Mayer waves reduce the accuracy of estimated hemodynamic response functions in functional near-infrared spectroscopy, Biomed Opt Express. 7 (2016) 3078-3088. 10.1364/boe.7.003078.

119. A. Savitzky, M.J.E. Golay, Smoothing and Differentiation of Data by Simplified Least Squares Procedures, Anal. Chem. 36 (1964) 1627-1639. 10.1021/ac60214a047. 
120. M.D. Pfeifer, F. Scholkmann, R. Labruyere, Signal Processing in Functional Near-Infrared Spectroscopy (fNIRS): Methodological Differences Lead to Different Statistical Results, Front Hum Neurosci. 11 (2017) 641. 10.3389/fnhum.2017.00641.

121. A. Vrana, M.L. Meier, S. Hotz-Boendermaker, B.K. Humphreys, F. Scholkmann, Cortical Sensorimotor Processing of Painful Pressure in Patients with Chronic Lower Back Pain-An Optical Neuroimaging Study using fNIRS, Front Hum Neurosci. 10 (2016) 578. 10.3389/fnhum.2016.00578.

122. A. Vrana, M.L. Meier, S. Hotz-Boendermaker, B.K. Humphreys, F. Scholkmann, Different mechanosensory stimulations of the lower back elicit specific changes in hemodynamics and oxygenation in cortical sensorimotor areas-A fNIRS study, Brain Behav. 6 (2016) e00575. 10.1002/brb3.575.

123. E. Kirilina, A. Jelzow, A. Heine, M. Niessing, H. Wabnitz, R. Bruhl, et al., The physiological origin of task-evoked systemic artefacts in functional near infrared spectroscopy, Neuroimage. 61 (2012) 70-81. 10.1016/j.neuroimage.2012.02.074.

124. T. Takahashi, Y. Takikawa, R. Kawagoe, S. Shibuya, T. Iwano, S. Kitazawa, Influence of skin blood flow on near-infrared spectroscopy signals measured on the forehead during a verbal fluency task, Neuroimage. 57 (2011) 991-1002. 10.1016/j.neuroimage.2011.05.012.

125. L. Duan, Z. Zhao, Y. Lin, X. Wu, Y. Luo, P. Xu, Wavelet-based method for removing global physiological noise in functional near-infrared spectroscopy, Biomed Opt Express. 9 (2018) 3805-3820. 10.1364/boe.9.003805.

126. M.A. Yucel, J.J. Selb, T.J. Huppert, M.A. Franceschini, D.A. Boas, Functional Near Infrared Spectroscopy: Enabling Routine Functional Brain Imaging, Curr Opin Biomed Eng. 4 (2017) 7886. 10.1016/j.cobme.2017.09.011.

127. H. Santosa, A. Aarabi, S.B. Perlman, T.J. Huppert, Characterization and correction of the falsediscovery rates in resting state connectivity using functional near-infrared spectroscopy, J Biomed Opt. 22 (2017) 55002. 10.1117/1.Jbo.22.5.055002. 
128. M. Caldwell, F. Scholkmann, U. Wolf, M. Wolf, C. Elwell, I. Tachtsidis, Modelling confounding effects from extracerebral contamination and systemic factors on functional near-infrared spectroscopy, Neuroimage. 143 (2016) 91-105. 10.1016/j.neuroimage.2016.08.058.

129. M. Cope, D.T. Delpy, E.O.R. Reynolds, S. Wray, J. Wyatt, P. van der Zee, Methods of Quantitating Cerebral Near Infrared Spectroscopy Data, in: M. Mochizuki, et al. (Eds.), Oxygen Transport to Tissue, Springer US: Boston, MA, 1988,. pp. 183-189.

130. D.T. Delpy, M. Cope, P. van der Zee, S. Arridge, S. Wray, J. Wyatt, Estimation of optical pathlength through tissue from direct time of flight measurement, Phys Med Biol. 33 (1988) 1433-1442. 10.1088/0031-9155/33/12/008.

131. T. Talukdar, J.H. Moore, S.G. Diamond, Continuous correction of differential path length factor in near-infrared spectroscopy, J Biomed Opt. 18 (2013) 56001. 10.1117/1.Jbo.18.5.056001.

132. P.-H. Chou, T.-H. Lan, The role of near-infrared spectroscopy in Alzheimer's disease, Journal of Clinical Gerontology and Geriatrics. 4 (2013) 33-36. 10.1016/j.jcgg.2013.01.002.

133. P. Ekkekakis, Illuminating the black box: investigating prefrontal cortical hemodynamics during exercise with near-infrared spectroscopy, J Sport Exerc Psychol. 31 (2009) 505-553. 10.1123/jsep.31.4.505.

134. F. Scholkmann, M. Wolf, General equation for the differential pathlength factor of the frontal human head depending on wavelength and age, J Biomed Opt. 18 (2013) 105004. 10.1117/1.Jbo.18.10.105004.

135. G. Strangman, M.A. Franceschini, D.A. Boas, Factors affecting the accuracy of near-infrared spectroscopy concentration calculations for focal changes in oxygenation parameters, Neuroimage. 18 (2003) 865-879. 10.1016/s1053-8119(03)00021-1.

136. A. Duncan, J.H. Meek, M. Clemence, C.E. Elwell, P. Fallon, L. Tyszczuk, et al., Measurement of cranial optical path length as a function of age using phase resolved near infrared spectroscopy, Pediatr Res. 39 (1996) 889-894. 10.1203/00006450-199605000-00025. 
137. M. Essenpreis, C.E. Elwell, M. Cope, P. van der Zee, S.R. Arridge, D.T. Delpy, Spectral dependence of temporal point spread functions in human tissues, Appl Opt. 32 (1993) 418425. 10.1364/ao.32.000418.

138. K. Nakamura, K. Kurihara, H. Kawaguchi, T. Obata, H. Ito, E. Okada, Estimation of partial optical path length in the brain in subject-specific head models for near-infrared spectroscopy, Opt Rev. 23 ( 2016) 316-322. 10.1007/s10043-016-0179-9.

139. M. Izzetoglu, R. Holtzer, Effects of Processing Methods on fNIRS Signals Assessed During Active Walking Tasks in Older Adults, IEEE Trans Neural Syst Rehabil Eng. 12 (2020) http://dx.doi.org/10.1109/TNSRE.2020.2970407.

140. H.J. Niu, X. Li, Y.J. Chen, C. Ma, J.Y. Zhang, Z.J. Zhang, Reduced frontal activation during a working memory task in mild cognitive impairment: a non-invasive near-infrared spectroscopy study, CNS Neurosci Ther. 19 (2013) 125-131. 10.1111/cns.12046.

141. J.D. Schaeffer, A.S. Yennu, K.C. Gandy, F. Tian, H. Liu, H. Park, An fNIRS investigation of associative recognition in the prefrontal cortex with a rapid event-related design, $\mathrm{J}$ Neurosci Methods. 235 (2014) 308-315. 10.1016/j.jneumeth.2014.07.011.

142. F.B. Haeussinger, T. Dresler, S. Heinzel, M. Schecklmann, A.J. Fallgatter, A.C. Ehlis, Reconstructing functional near-infrared spectroscopy (fNIRS) signals impaired by extra-cranial confounds: an easy-to-use filter method, Neuroimage. 95 (2014) 69-79. 10.1016/j.neuroimage.2014.02.035.

143. T.J. Huppert, R.D. Hoge, S.G. Diamond, M.A. Franceschini, D.A. Boas, A temporal comparison of BOLD, ASL, and NIRS hemodynamic responses to motor stimuli in adult humans, Neuroimage. 29 (2006) 368-382. 10.1016/j.neuroimage.2005.08.065.

144. X. Cui, S. Bray, A.L. Reiss, Functional near infrared spectroscopy (NIRS) signal improvement based on negative correlation between oxygenated and deoxygenated hemoglobin dynamics, Neuroimage. 49 (2010) 3039-3046. 10.1016/j.neuroimage.2009.11.050. 
145. G. Allali, H.M. Blumen, H. Devanne, E. Pirondini, A. Delval, D. Van De Ville, Brain imaging of locomotion in neurological conditions, Neurophysiol Clin. 48 (2018) 337-359. http://dx.doi.org/10.1016/j.neucli.2018.10.004.

146. M. Ferrari, S. Bisconti, M. Spezialetti, S. Basso Moro, C. Di Palo, G. Placidi, et al., Prefrontal cortex activated bilaterally by a tilt board balance task: a functional near-infrared spectroscopy study in a semi-immersive virtual reality environment, Brain Topogr. 27 (2014) 353-365. 10.1007/s10548-013-0320-z.

147. D.J. Clark, D.K. Rose, S.A. Ring, E.C. Porges, Utilization of central nervous system resources for preparation and performance of complex walking tasks in older adults, Front Aging Neurosci. 6 (2014) http://dx.doi.org/10.3389/fnagi.2014.00217.

148. T. Huppert, J. Barker, B. Schmidt, S. Walls, A. Ghuman, Comparison of group-level, source localized activity for simultaneous functional near-infrared spectroscopymagnetoencephalography and simultaneous fNIRS-fMRI during parametric median nerve stimulation, Neurophotonics. 4 (2017) 015001. 10.1117/1.NPh.4.1.015001.

149. H. Sato, N. Yahata, T. Funane, R. Takizawa, T. Katura, H. Atsumori, et al., A NIRS-fMRI investigation of prefrontal cortex activity during a working memory task, Neuroimage. 83 (2013) 158-173. 10.1016/j.neuroimage.2013.06.043.

150. V. Scarapicchia, C. Brown, C. Mayo, J.R. Gawryluk, Functional Magnetic Resonance Imaging and Functional Near-Infrared Spectroscopy: Insights from Combined Recording Studies, Front Hum Neurosci. 11 (2017) 419. 10.3389/fnhum.2017.00419.

151. J.A. Noah, Y. Ono, Y. Nomoto, S. Shimada, A. Tachibana, X. Zhang, et al., fMRI Validation of fNIRS Measurements During a Naturalistic Task, J Vis Exp. (2015) e52116. 10.3791/52116.

152. A. Berger, F. Horst, F. Steinberg, F. Thomas, C. Muller-Eising, W.I. Schollhorn, et al., Increased gait variability during robot-assisted walking is accompanied by increased sensorimotor brain activity in healthy people, J Neuroeng Rehabil. 16 (2019) http://dx.doi.org/10.1186/s12984019-0636-3. 
153. M. Muthalib, A.R. Anwar, S. Perrey, M. Dat, A. Galka, S. Wolff, et al., Multimodal integration of fNIRS, fMRI and EEG neuroimaging, Clin Neurophysiol. 124 (2013) 2060-2062. 10.1016/j.clinph.2013.03.018.

154. R. Trevethan, Sensitivity, Specificity, and Predictive Values: Foundations, Pliabilities, and Pitfalls in Research and Practice, Front Public Health. 5 (2017) 307. 10.3389/fpubh.2017.00307.

155. S.J. Colcombe, A.F. Kramer, K.I. Erickson, P. Scalf, The implications of cortical recruitment and brain morphology for individual differences in inhibitory function in aging humans, Psychol Aging. 20 (2005) 363-375. 10.1037/0882-7974.20.3.363.

156. R. Cabeza, N.D. Anderson, J.K. Locantore, A.R. McIntosh, Aging gracefully: compensatory brain activity in high-performing older adults, Neuroimage. 17 (2002) 1394-1402. 10.1006/nimg.2002.1280.

157. P.A. Reuter-Lorenz, K.A. Cappell, Neurocognitive aging and the compensation hypothesis. , Curr Dir Psychol Sci. 17 (2008) 177-182.

158. Y. Bhambhani, R. Maikala, M. Farag, G. Rowland, Reliability of near-infrared spectroscopy measures of cerebral oxygenation and blood volume during handgrip exercise in nondisabled and traumatic brain-injured subjects, J Rehabil Res Dev. 43 (2006) 845-856. 10.1682/jrrd.2005.09.0151.

159. M.M. Plichta, M.J. Herrmann, C.G. Baehne, A.C. Ehlis, M.M. Richter, P. Pauli, et al., Eventrelated functional near-infrared spectroscopy (fNIRS): are the measurements reliable?, Neuroimage. 31 (2006) 116-124. 10.1016/j.neuroimage.2005.12.008.

160. D. Tsuzuki, I. Dan, Spatial registration for functional near-infrared spectroscopy: from channel position on the scalp to cortical location in individual and group analyses, Neuroimage. $85 \mathrm{Pt}$ 1 (2014) 92-103. 10.1016/j.neuroimage.2013.07.025.

161. S. Perrey, P. Besson, Studying brain activity in sports performance: Contributions and issues, Prog Brain Res. 240 (2018) 247-267. 10.1016/bs.pbr.2018.07.004. 
162. R.F. Rojas, X. Huang, K.-L. Ou, Region of Interest Detection and Evaluation in Functional near Infrared Spectroscopy, J. Near Infrared Spectrosc. 24 (2016) 317-326.

163. J.U. Blicher, C.J. Stagg, J. O'Shea, L. Ostergaard, B.J. Maclntosh, H. Johansen-Berg, et al., Visualization of altered neurovascular coupling in chronic stroke patients using multimodal functional MRI, J Cereb Blood Flow Metab. 32 (2012) 2044-2054. 10.1038/jcbfm.2012.105.

164. A.S. Salinet, N.C. Silva, J. Caldas, D.S. de Azevedo, M. de-Lima-Oliveira, R.C. Nogueira, et al., Impaired cerebral autoregulation and neurovascular coupling in middle cerebral artery stroke: Influence of severity?, J Cereb Blood Flow Metab. 39 (2019) 2277-2285. $10.1177 / 0271678 \times 18794835$.

165. L. Wang, H. Ayaz, M. Izzetoglu, Investigation of the source-detector separation in near infrared spectroscopy for healthy and clinical applications, J Biophotonics. 12 (2019) e201900175. 10.1002/jbio.201900175.

166. M.D. Fox, Mapping Symptoms to Brain Networks with the Human Connectome, N Engl J Med. 379 (2018) 2237-2245. 10.1056/NEJMra1706158.

167. B. Wang, M. Zhang, L. Bu, L. Xu, W. Wang, Z. Li, Posture-related changes in brain functional connectivity as assessed by wavelet phase coherence of NIRS signals in elderly subjects, Behav Brain Res. 312 (2016) 238-245. 10.1016/j.bbr.2016.06.037.

168. L.M. Hocke, I.K. Oni, C.C. Duszynski, A.V. Corrigan, B.D. Frederick, J.F. Dunn, Automated Processing of fNIRS Data-A Visual Guide to the Pitfalls and Consequences, Algorithms. 11 (2018) 10.3390/a11050067.

169. R. Holtzer, C. Schoen, E. Demetriou, J.R. Mahoney, M. Izzetoglu, C. Wanget al., Stress and gender effects on prefrontal cortex oxygenation levels assessed during single and dual-task walking conditions, Eur J Neurosci. 45 (2017) 660-670. 10.1111/ejn.13518.

170. R. Holtzer, J. Verghese, G. Allali, M. Izzetoglu, C. Wang, J.R. Mahoney, Neurological Gait Abnormalities Moderate the Functional Brain Signature of the Posture First Hypothesis, Brain Topogr. 29 (2016) 334-343. 10.1007/s10548-015-0465-z. 
171. R. Holtzer, J. Yuan, J. Verghese, J.R. Mahoney, M. Izzetoglu, C. Wang, Interactions of Subjective and Objective Measures of Fatigue Defined in the Context of Brain Control of Locomotion, J Gerontol A Biol Sci Med Sci. 72 (2017) 417-423. 10.1093/gerona/glw167.

172. M. Lucas, M.E. Wagshul, M. Izzetoglu, R. Holtzer, Moderating Effect of White Matter Integrity on Brain Activation During Dual-Task Walking in Older Adults, J Gerontol A Biol Sci Med Sci. 74 (2019) 435-441. http://dx.doi.org/10.1093/gerona/gly131.

173. R. Holtzer, R. Kraut, M. Izzetoglu, K. Ye, The effect of fear of falling on prefrontal cortex activation and efficiency during walking in older adults, GeroScience. 41 (2019) 89-100. http://dx.doi.org/10.1007/s11357-019-00056-4.

174. X.N. Zuo, J.S. Anderson, P. Bellec, R.M. Birn, B.B. Biswal, J. Blautzik, et al., An open science resource for establishing reliability and reproducibility in functional connectomics, Sci Data. 1 (2014) 140049. 10.1038/sdata.2014.49.

175. C.L. Tardif, A. Schafer, R. Trampel, A. Villringer, R. Turner, P.L. Bazin, Open Science CBS Neuroimaging Repository: Sharing ultra-high-field MR images of the brain, Neuroimage. 124 (2016) 1143-1148. 10.1016/j.neuroimage.2015.08.042. 


\section{TABLES}

Table 1. Summary of key point recommendations and considerations 


\section{FIGURES}

Figure 1. Examples of block design (A) and event-related design (B) used in fNIRS studies of posture and gait. The interval of reference distinguishes between designs.

A) Block design: the concentration in oxygenated haemoglobin ( $\mathrm{HbO2}$ ) during a balance / gait task (0s to 20 s, here) is normalised to a static baseline (-10 to 0 s, here) immediately preceding the onset of the task of interest. The zero crossing indicates the start of the actual task condition (adapted from Mirelman et al., 2014) [9].

B) Event-related design: the concentration in oxygenated haemoglobin ( $\mathrm{HbO2}$ ) during an "event", for example, a turn (blue trace) or a freezing of gait (FOG) event as displayed here, is normalised to a dynamic baseline, here normal walking (green trace) (adapted from Maidan et al., 2015) [32].

Figure 2. Summary of fNIRS data processing steps.

Figure 3. Examples of different levels of filtering on $\mathrm{HbO2}$ signal acquired from prefrontal cortex channels during: (A) 20 stepping trials of inhibitory stepping test; (B) walking. Note how the addition of other filters (wavelet with or without CBSI filters) attenuates the signal. 
SUPPLEMENTARY MATERIALS

Table S1. Checklist of items to consider at processing and reporting steps of fNIRS data collected in studies of posture and gait. 

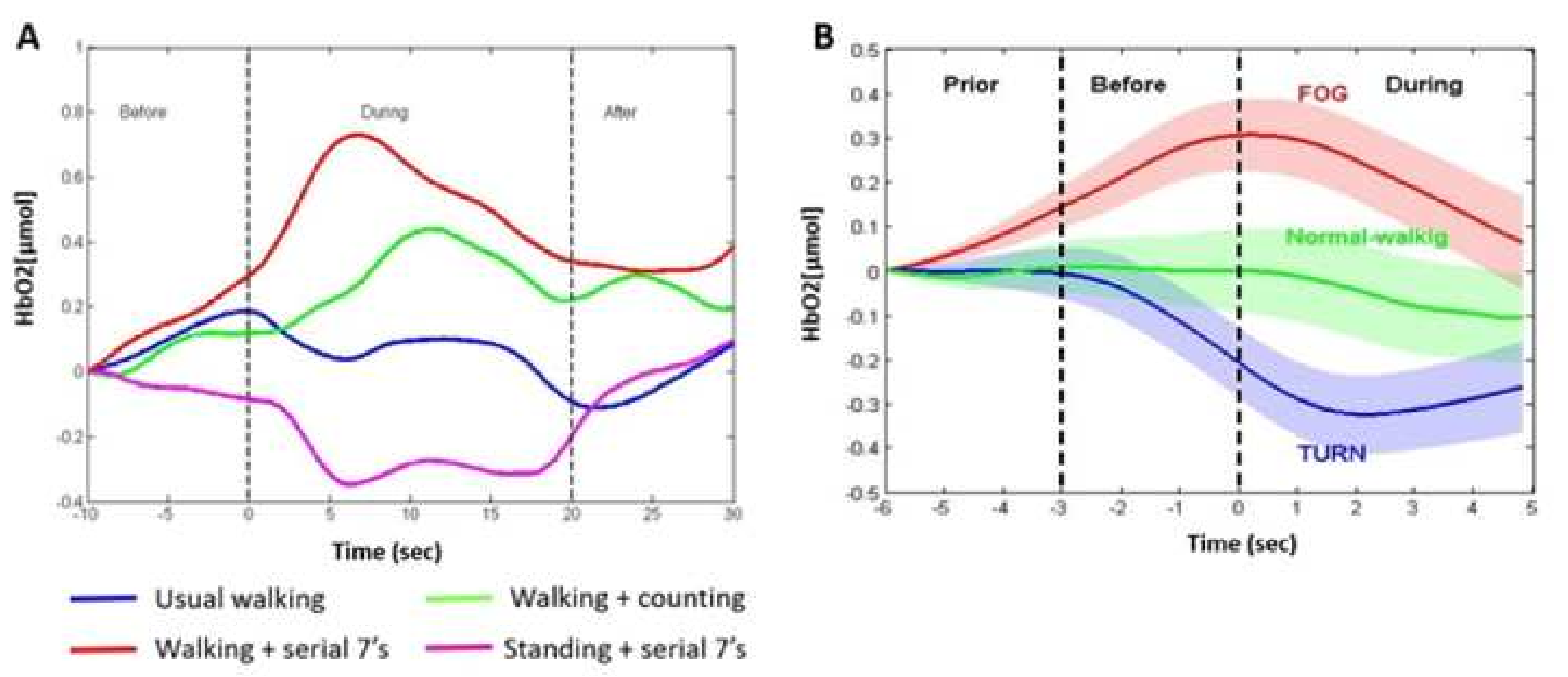
- Check the data quality

> Perform a visual inspection of the raw data and exclude channels with insufficient data quality based on objective criterions (e.g., distinct coefficient of variation).

- Removal of motion artefacts (on the optical density measurements)

> Use of high-performance methods taking into account the structure of movement-related noise (e.g., low frequency components associated with postural sway or high vertical accelerations associated with foot impacts when walking)

- Conversion of optical density changes into concentration changes of chromophores (e.g. $\mathrm{HbO2,} \mathrm{Hhb,} \mathrm{total} \mathrm{Hb}$ )

$>$ Apply the modified Beer-Lambert law with appropriate $\mu$ a and DPF values (i.e. wavelength- and age-dependent DPF)

- Removal of physiological artefacts and other sources of noise

$>$ Use of high-performing methods (e.g. short-channel regression to filter out extracerebral signal components)

$>$ Use a Band-pass filtering with appropriate cut-off frequencies (e.g. considering stimulus and/or task paradigm)

- Perform a detrending

> Compare experimental signal to baseline signal (block- or eventrelated design) 

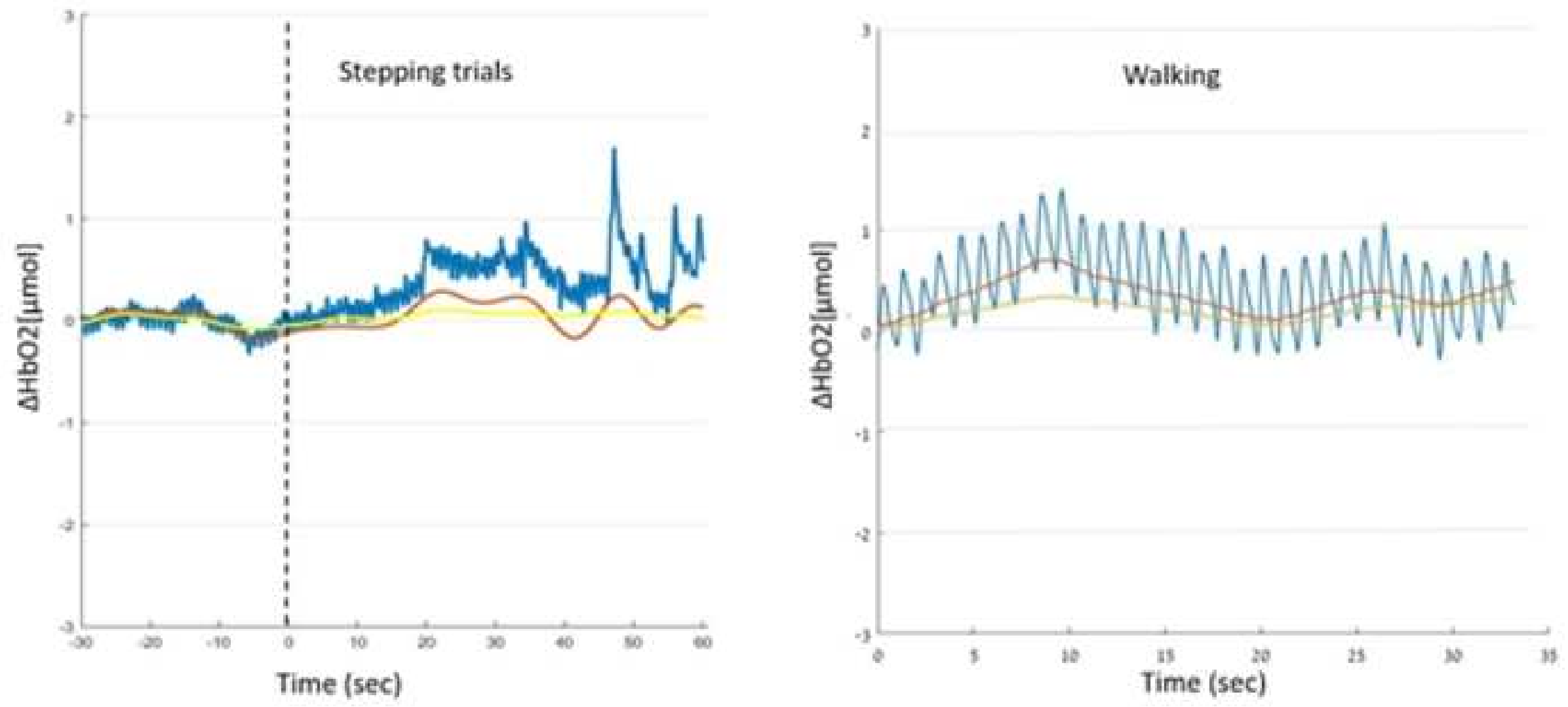

Raw Band-pass filter

Band-pass filter + Wavelet transform (+ CBSI filters (left)) 
Table 1. Summary of key point recommendations and considerations

Hardware set-up and study protocols

- Consider cap stretch effect on inter-optode distance

- Consider chinstraps effect on data in verbal tasks (e. dual tasks)

- Consider optimal optode design for study's goals, data quality versus participants' comfort

- Detail methods used for optode positions relative to cortical anatomy

A-priori control of confounding factors and post data acquisition processing of artefacts

- Outline processing steps and assumptions made regarding:

- Ensuring adequate signal-to noise ratio

- Control of confounding factors a-priori: environment, instrument, motion and physiology-related

- Data quality checks post-acquisition and removal of channels with insufficient quality

- Removal of motion artefacts

- Correction for physiology-related artefacts

- Consideration of differential path length factor assumptions

- Report amount of excluded data and reasons in detail

- Describe the software and specific processing pipelines used

- Ensure accurate synchronization with external devices

Outcome measures, validity and reliability 
- Report both $\mathrm{HbO} 2$ and $\mathrm{HHb}$ outcomes and assess the strength of their correlation

- Consider potential effect of asymmetrical pathologies on hemodynamics

- Report on test-retest reliability of specific tasks for both $\mathrm{HbO} 2$ and $\mathrm{HHb}$

- Consider learning effects of the task(s) on hemodynamics

Transparency in reporting, data sharing

- Provide a clear definition of the regions of interest and justification of associated channels

- For clinical groups: describe brain lesions and proximity to fNIRS channels

- Devise an a-priori approach to data removal and report missing data

- Consider data sharing through open science repositories 


\section{Conflict of Interest}

Please find below the list of authors together with any conflict of interest they declare.

\begin{tabular}{|l|l|}
\hline Author & Conflict of Interest \\
\hline Jasmine C. Menant & None \\
\hline Inbal Maidan & None \\
\hline Lisa Alcock & None \\
\hline Emad Al-Yahya & None \\
\hline Antonio Cerasa & None \\
\hline David J. Clark & None \\
\hline Eling de Bruin & None \\
\hline Sarah Fraser & None \\
\hline Vera Gramigna & None \\
\hline Dennis Hamacher & None \\
\hline Fabian Herold & None \\
\hline Roee Holtzer & None \\
\hline Meltem Izzetoglu & Dr. Izzetoglu has a very minor share in the company, fNIR Devices, LLC, \\
\hline Photomac, MD that manufactures fNIRS devices \\
\hline Annette Pantall & None \\
\hline Paulo Pelicioni & None \\
\hline Sue Peters & None \\
\hline Andrea L. Rosso & None \\
\hline Rebecca St George & None \\
\hline Samuel Stuart & None \\
\hline Roberta Vasta & None \\
\hline Rodrigo Vitorio & None \\
\hline Anat Mirelman & None \\
\hline & \\
\hline
\end{tabular}


Table S1

Click here to access/download Supplementary Material

Table S1.docx 


\begin{abstract}
Background: Functional near-infrared spectroscopy (fNIRS) is increasingly used in the field of posture and gait to investigate patterns of cortical brain activation while people move freely. fNIRS methods, analysis and reporting of data vary greatly across studies which in turn can limit the replication of research, interpretation of findings and comparison across works.
\end{abstract}

Research question and methods: Considering these issues, we propose a set of practical recommendations for the conduct and reporting of fNIRS studies in posture and gait, acknowledging specific challenges related to clinical groups with posture and gait disorders. Results: Our paper is organized around three main sections: 1) hardware set up and study protocols, 2) artefact removal and data processing and, 3) outcome measures, validity and reliability; it is supplemented with a detailed checklist.

Significance: This paper was written by a core group of members of the International Society for Posture and Gait Research and posture and gait researchers, all experienced in fNIRS research, with the intent of assisting the research community to lead innovative and impactful fNIRS studies in the field of posture and gait, whilst ensuring standardization of research.

Keywords: functional-Near Infrared Spectroscopy; guidelines: cerebral hemodynamics; posture; gait; balance.

Funding: This research did not receive any specific grant from funding agencies in the public, commercial or not-for-profit sectors. 
Declarations of interest: None

\section{Introduction}

Functional near-infrared spectroscopy (fNIRS) is an optical neuroimaging technique that monitors hemodynamic responses in superficial cortical regions. The fNIRS raw data extracted from most devices is light intensity. Through computation of the differential light intensity between the input and output, these data can then be converted to represent changes in the concentration of oxygenated and deoxygenated hemoglobin ( $\mathrm{HbO} 2$ and $\mathrm{HHb}$, respectively) across all vascular compartments (arteries, veins and capillaries) [1]. The neurovascular coupling process enables these $\mathrm{HbO} 2$ and $\mathrm{Hhb}$ concentration changes to be considered as surrogates for neural activation [2-4]. The fNIRS technique has revolutionised the field of posture and gait largely due to its portability; the ability to assess brain activation during actual task performance (i.e., walking, balancing). As such, it addresses a key limitation of other commonly used neuroimaging techniques such as functional magnetic resonance imaging, which involves static tasks and/or supine posture in order to minimize movement.

The increasing availability of commercial fNIRS devices has facilitated the extensive use of this technique to investigate cortical contributions to gait and postural control. fNIRS has been used to explore questions relating to cortical activation during balance tasks (e.g. [5-10]), stepping tasks (e.g. $[6,11])$, walking over unobstructed paths (e.g. $[12,13])$ or paths with obstacles (e.g. [14-17]), treadmill walking (e.g. [18-24]) and walking with and without concurrently performing secondary cognitive (e.g. [12, 25-30]) or motor tasks (e.g. [31]). The majority of studies focused on young and older adults (e.g. [12, 23, 24, 28, 30, 32, 33]), but some research has involved clinical populations (e.g., Parkinson's disease (e.g. [34-41]), stroke 
(e.g. [17, 42-48]), multiple sclerosis (e.g. [49-52]). Areas of interest have primarily covered the prefrontal cortex (e.g. $[12,20,31,53])$, the pre-supplementary motor area (e.g. [20]), the supplementary motor area (e.g. [20,31]), the premotor cortex (e.g. $[6,7,32,33])$, the primary motor cortex (e.g. $[6,7,20])$, the sensorimotor cortex (e.g. $[20,33])$, the superior temporal gyrus (e.g. [5]) and all superficial cortical areas that the near-infrared light can penetrate. The results of the published studies have increased our understanding of the cortical involvement in gait and postural control and can be interpreted in the context of theories relating to neural compensation, inefficiency and capacity [54]. These theories relate to either the increase in neural activation efforts to maintain performance despite declining brain capacity (also known as "less wiring, more firing") [55-57] or the capacity limitation model which suggests that a reduction in activation is synonymous to limited brain resources resulting in poor performance on one or both tasks.

The increasing number of studies using fNIRS in balance and gait research is demonstrated by the rising number of published systematic reviews, $>15$ published in the past 10 years (e.g., [58-72]). Yet from these reviews, it is apparent that the obvious benefits related to knowledge growth are hampered by the inconsistency and lack of details in the reporting of experimental and data analysis protocols. This significantly limits the replication of research, its interpretation in a wider context and comparison across works. Aside from practical points and take-home messages provided in the conclusions of reviews, guidelines regarding the reporting of fNIRS data in posture and gait research do not exist. In view of these concerns, the goal of this consensus paper is to summarize the current state of knowledge on the use of fNIRS for the study of posture and gait and identify knowledge gaps that offer high probability of leading to innovations in the field. The paper is divided into three main sections: 
1) hardware set up and study protocols, 2) artefact removal and data processing and 3) outcome measures, validity and reliability.

\section{Hardware set up and study protocols}

Many different fNIRS devices and configurations have been used in the field of posture and gait, including custom-made and commercially available units. Some systems offer single channels to measure from specific regions of interest (ROIs) while others offer many channels covering broader areas of the scalp, both have advantages and limitations $[73,74]$. Multichannel units present the obvious benefit of recording from more cortical regions in a single recording session, but also suffer from lower sampling rates as a result of signal multiplexing needed to distinguish between channels [73]. This can have an adverse impact on data quality because low sampling rates preclude the ability to apply some of the recommended signal processing steps. Single channels on the other hand focus on a single ROI, which in complex functions such as gait and balance may limit our understanding of the network of regions involved and important changes across regions that may occur with different task demands or in response to interventions. Ultimately, the choice of fNIRS device should be motivated by the specific research questions.

Because of the comparative nature of the fNIRS technique, hemodynamic changes can be explored in an event-related or block design (Figure 1). In both cases, recording needs to be of sufficient duration to observe the onset (about 1-2 seconds after neural firing) and peak (about 4-7 seconds) of the hemodynamic response [75]. Block designs are generally appropriate to measure both transient and sustained cortical activity related to experimental tasks involving prolonged continuous, reciprocal movements. Walking and steady state 
standing are good examples. In block design trials, baseline periods following experimental task periods should be sufficient for the hemodynamic response to return towards its original baseline levels. It is important to consider that for block design paradigms with as little as four repetitions, anticipatory responses may occur [32]. This can be controlled for by varying baseline intervals so that the onset of the experimental task is difficult to predict or use a specific section within the middle of each block. There is currently no gold standard for the number of trials required to reduce variability of fNIRS signal $[61,68,70,72]$. Nevertheless, using at least three trials will allow averaging over several fNIRS signals and should minimize anticipatory contributions.

Event-related designs tend to be more suited to measuring cortical activity in response to acute events, such as gait initiation, postural reactions to balance perturbations, and specific gait phenomena such as freezing of gait, turns or obstacle negotiation (e.g. $[6,11,16,35])$. In such a design, it is crucial to synchronize the event with the fNIRS signals. To capture the hemodynamic response, the protocol should be designed to record at least 3 seconds of the time: before the event, during the event and after the event; this will enable to capture the peak of the response for a single stimulus. For event-related designs, shorter baselines will allow significantly more trials to do more powerful statistics [76]. Conversely, it is also important to consider appropriate inter-stimulus interval which, if too brief, will cause the event-related responses to overlap, in turn compromising the nature of the event-related design. This event-related method allows investigating individual response to a stimulus but poses a challenge when compared within or between groups due to the potential betweensubjects variance in hemodynamic response. It is thus essential for researchers to detail the experimental procedure and account for differences between subjects where applicable. 
These inherent limitations of fNIRS methodology should be considered carefully in protocol design. An emphasis should be placed on selecting an appropriate baseline for the task studied. Since posture and gait studies are conducted upright, baseline fNIRS recordings have to be in upright position to eliminate changes due to gravitational blood pressure fluctuations [77].

\section{Optode placement}

To ensure scientific rigor and reproducibility, optode placement on the scalp should be reported relative to anatomical landmarks. The common approach is to use the international 10-20 system, which defines scalp locations as a percentage of the individual's head size [78]. Initial measurements include mid-sagittal plane distance (nasion to inion), a frontal plane distance (left to right pre-auricular point), and head circumference. Ideally, in the case of customizable optode arrays, specific standardized scalp locations should be determined based on percentages of those initial measurements. Given the obvious ambiguity in localizing surface anatomy landmarks (e.g. peri-auricular points and inion)[79], explicitly defining landmark locations is important for maintaining consistent landmarking optode locations across sessions.

A key concern to any fNIRS research study is to ensure that the optode location effectively
targets the selected underlying cortical ROI. The Gold standard method is to obtain a recent
structural Magnetic Resonance Imaging (MRI) scan of the individual's brain and co-register
the digitized optode locations on the scalp with the underlying cortical site(s). Yet the costs
and logistics associated with brain MRI data collection can be a major obstacle. In the absence
of brain MRI scans, the fNIRS Optodes Location Decider (fOLD) approach and the use of 3D 
digitization are available to guide the selection of optode positions for fNIRS experiments [80]. The fOLD method is based on photon transport simulations on two head atlases and the toolbox is freely available for download (Table S1). The 3D digitizing method allows to project optode locations onto brain atlases [81]. The translation of optodes positioning to precise cortical ROls remains a challenge because there can be considerable variability in brain morphology among individuals. In particular, existing neuroimaging research on brain morphology has identified large variation in older adults and people with brain pathologies such as stroke, traumatic brain injury, or neurodegeneration $[82,83]$. This should be taken into consideration when evaluating between-subject designs.

In within-subjects designs, a convenient way to improve consistency is to supplement $10-20$ land marking with digitization of the optode using a 3D digitizing pen. Differences between optode locations across multiple testing sessions can then be calculated to determine the variance in optode placement [84]. If the estimated optode location has a large difference between sessions (i.e. greater than the inter-optode distance), the following options should be taken: 1) discard the optode from multi-session comparisons, 2) determine if another optode was set up closer to the optode of interest.

\section{Caps, hair, scalp and chinstraps considerations}

Optodes are typically held in place by a cap or headband. Most caps are flexible and often come with pre-cut holes (some corresponding to 10-20 landmarks) hence allowing for customizable optode arrays. However, variation in the relative stretch of the cap over different scalp areas or between participants can alter the inter-optode distance, affect signal intensity, and introduce variability in inter-subject optode locations. 
Optodes with a pointed tip might be required when the desired optode location is covered by hair. However, this might increase noise level relative to the signal. Further, the pointed-tip optode design is likely to increase pressure at optode locations, in order to maximize contact with the scalp. The increased pressure may further impact skin blood flow which can increase superficial layer contamination in fNIRS measurements. The pressure from the optodes may also cause discomfort for the participant. In this situation, the recorded cortical activity could be biased by attention to the discomfort and further limit the tolerable duration of the testing time. Strategies to manage this issue include keeping data collection sessions short and/or taking extra time to separate the hair beneath each optode such that tightening of the cap can be minimized to avoid discomfort for the participant.

If a chinstrap is used to secure the cap in place, it can increase the risk of talking-induced movement artefacts $[85,86]$. This is particularly important for studies that include tasks requiring vocal response, such as in dual-task paradigms that pair walking or balance with a verbal cognitive task. Headband configuration units are less influenced by verbal responses, however, measurements are limited to the prefrontal cortex. In some systems the optode configurations are adjustable while in other they are fixed in place, which limits flexibility of the array but ensures consistent inter-optode distance and improves optode placement uniformity across participants. Differences in brain morphology may influence the signal and interpretation, therefore, they should be reported and taken into consideration during analysis. Future consensus efforts should be made by posture and gait researchers to achieve standardisation of optode positioning through the establishment of brain fNIRS-MRI repositories. 


\section{Artefact removal and data processing}

fNIRS signals are influenced by a variety of confounding factors that should be controlled for to optimize data quality. fNIRS data should be recorded with an adequate signal-to-noise ratio reflected in a close coupling of the optodes with the scalp. A few checks can be used to ensure good data quality prior to data acquisition: (i) heart rate oscillations clearly visible in each channel [87]; (ii) channel-wise metrics set-up by the manufacturers and which rely, for instance, on the calculation of the coefficient of variation to rate signal quality (Table S1); (iii) use of freely available software 'PHOEBE' which detects cardiac pulsation automatically and can be used to adjust and ensure a relative optimal optode-scalp coupling [88]. This section reviews common confounding factors and methodologies used in the posture and gait field to account for them. Figure 2 provides a summary of the fNIRS data processing steps.

\section{Environmental conditions}

The environmental conditions of laboratory settings (e.g. room temperature, humidity, sound, light) should be kept stable to ensure that the electronic devices perform optimally and that the participants do not experience discomfort. For example heat stress would influence the cardiorespiratory system, inducing systemic physiological changes (e.g. increased heart rate and blood flow) which may confound the fNIRS signal and lead to 'false positive' findings $[89,90]$. Sweating is also likely to affect light sources and detector coupling with the skin. Loud sounds could also affect chromophore concentration through attentional interference, as seen in functional MRI experiments [91]. It is also recommended to conduct the experiments in a room with dimmed lights and/or to use a dark head cap to cover and 
shield the optodes from ambient light [89] as light, including variations in colored light, has been found to contaminate signals [92-94].

\section{Instrument-related artefacts}

Instrumental configurations such as wavelength selection, measurement frequency and type of light detectors can influence the signal quality, however, they cannot be easily changed by the user. Hence, the importance of carefully reporting them in sufficient detail and following the manufacturers' instructions. With regard to the illumination source, lasers require some heating time to perform optimally; thus it is recommended that the instrumentation be switched on with some time before starting fNIRS data acquisition [89]. To reduce cross-talk (e.g. incorrect separation of changes in $\mathrm{HbO} 2$ and $\mathrm{HHb}$ ) which heavily depends on the wavelength selection, an optimal combination of wavelengths should be used $[73,89]$. Even though there is currently no consensus as to which combination of wavelengths is optimal $[61,73]$, the degree of cross-talk has been deemed to be relatively minimal when using one wavelength $>730 \mathrm{~nm}$ and another $<720 \mathrm{~nm}$ [95]. Of note, commonly used commercial systems do not allow changing these parameters and typically report one wavelength between $705 \mathrm{~nm}$ and $760 \mathrm{~nm}$ and another around $850 \mathrm{~nm}$ [66].

\section{Motion-related artefacts}

In any balance and gait research, motion-related artefacts are unavoidable because of the movement involved in the execution of balance or walking tasks. Head motion might lead to changes in optode-scalp coupling which in turn, influences light detection [89]. It can further cause changes in the measured cortical location or shifts in cortical hemodynamic levels irrelevant of task related activations. These distinct effects can be reflected as different types 


\section{Physiology-related artefacts}

fNIRS signals not only record changes in cerebral hemodynamics but are also affected by variations in systemic physiology (e.g. fluctuations in heart rate, respiration, and/or blood pressure) [90]. These can increase the risk of finding 'false positives' because detected hemodynamic responses are wrongly attributed to functional brain activity. Thus, in order to elucidate the physiological origin of observed hemodynamic brain changes, it is possible to use multimodal physiological monitoring; an approach which has recently been termed 'systemic-physiology-augmented fNIRS' (SPA-fNIRS) neuroimaging [90, 93, 94]. This method applies short-separation channels to quantify systemic changes in the extracerebral layer [61, 
$70,90]$ and to remove skin response (the overall effect of extracerebral or superficial layers) from the long separation channels to obtain the cortical responses $[90,102,103]$. In addition, it is possible to capture changes in heart rate (e.g. via portable heart rate monitor or a pulse oximeter), blood pressure (e.g. based on pulse transit time), electrodermal activity (e.g. via skin conductance response) and respiration (e.g. via breathing rate and arterial partial pressure of carbon dioxide) $[93,94,104]$; the downside being over-instrumenting participants which may interfere with natural walking patterns.

\section{Post data acquisition processing}

To process and analyze fNIRS data, custom-written scripts, open-source toolboxes [96] or fNIRS manufacturers' software can be used (Table S1). However, regardless of which are utilized, processing information should be reported transparently and with sufficient detail to be replicated.

\section{Visual inspection and motion artefact removal}

As a first step, visual inspection of raw and/or relative optical density data is necessary to get an overview of data quality. Channels with insufficient data quality (see Table S1 for definitions) should then be removed. It is then advised to repeat the visual inspection to ensure that the exclusion algorithm has worked effectively. When using fNIRS in posture and gait, particular care needs to be taken to correct for motion-related artefacts. A large variety of methods are available [105] and can be classified as data-based approaches (e.g. using only fNIRS signals themselves) and approaches correcting for external biomechanical recordings. Among the variety of data-based approaches for removing motion artefacts (Table S1), spline interpolation [106], wavelet-based filters [107-110], or hybrid filter methods [111] are shown 
to be the most promising and powerful methods. To date, there is no consensus on the most effective filter methods to reduce motion artefacts in posture and gait tasks (e.g. low frequency components associated with postural sway, high vertical accelerations associated with foot strikes when walking). This is an important area for future fNIRS research.

\section{Correction of physiological artefacts and superficial layer contamination}

To correct for physiological artefacts, such as heart rate (0.5 to $2.0 \mathrm{~Hz}$ ), low-frequency components from blood pressure changes (Mayer waves) $(0.07$ to $0.13 \mathrm{~Hz}$ ) and respiration ( 0.2 to $0.4 \mathrm{~Hz})[73,90,105,112-115]$, a variety of filtering methods have been proposed (Table S1). High-pass and low-pass filters are commonly used to eliminate other sources of noise, but the applied cut-off frequencies should be chosen carefully in order to avoid the removal of stimulus-dependent hemodynamic responses $[61,104,116]$. The cut-off frequency of highpass filters is commonly set at $\sim 0.01 \mathrm{~Hz}$ to remove instrumental-related artefacts and vascular endothelial regulations $[117,118]$ and should be adopted for trials of extended durations (e.g. longer than 100s) [117]. Low-pass filters are commonly used to remove physiological oscillations (e.g., heart rate and/or Mayer waves). A cut-off frequency higher than the stimulus frequency and lower than the frequency of Mayer waves $(<0.1 \mathrm{~Hz})$ is recommended [117]. As alternative to bandpass filters, Savitzky-Golay filters [119] can be used for the purpose of smoothing the data, to increase the precision of the data without distorting the signal tendency. This is achieved, through convolution which can also be used in fNIRS studies [120-122]. Figure 3 provides examples of raw and filtered hemodynamic data.

In addition, the detected fNIRS signals contain both the cerebral hemodynamic activity (of interest) and also extracerebral hemodynamic activity originating from vascularized scalp and 
skull tissue $[90,123,124]$. Sympathetic activity and blood pressure changes associated with posture and gait tasks can result in changes that are not directly task-related. This may require the elimination of the extracerebral hemodynamic activity. Such activity can be filtered to an extent via techniques such as wavelet-based filtering or filters based on principal component analysis [125]. However, a more direct and recently commercially available method involves the application of short-separation channels $(0.5-1 \mathrm{~cm})$ which measure the extracerebral activity alone, so that it may be removed from the total fNIRS signal $[61,126]$. In this regard, it should be noted that the data quality of short-separation channels need to be acceptable, otherwise additional error is introduced [127]. While short-separation channels are a powerful tool to account for systemic physiological artefacts in fNIRS studies, many commercially available systems have fixed optode distances and do not allow for capturing short-separation channels. Approaches to deal with other systemic confounders (e.g., changes in blood pressure or arterial partial pressure of carbon dioxide) have been suggested [128], but have yet to be examined in studies investigating posture or gait [61].

\section{Consideration of the differential path length factor}

The differential path length factor (DPF) is a dimensionless correction factor used in the modified Beer-Lambert law to calculate the concentration of the chromophores (e.g. HbO2 and $\mathrm{HHb})[129,130]$. An inaccurately determined DPF can cause serious cross-talk error [131]. In the modified Beer-Lambert law, the DPF is needed to account for the scatter-dependent increase of optical path length occurring in biological tissue [132-135]. The DPF exhibits large inter-individual heterogeneity [134, 136-138] and is influenced by a variety of factors (see Table S1 for a list). It should be noted that ageing and pathology-related changes in DPF values (e.g. in Parkinson's disease or stroke) are not well-investigated and there is currently, to the 
best of our knowledge, no equation available to account for this. Hence, caution should be paid when comparing findings between groups entailing different pathologies [70]. Recent findings show block design protocols involving highly validated and reliable tasks (e.g. dualtask walking) might be robust to variations in conversion parameters (used in the BeerLambert law, including the DPF) and different low-pass filter applications [139]. Yet, to ensure data repeatability and comparison, it is important to report the parameter values used in conversion to $\mathrm{HbO} 2$ and $\mathrm{HHb}$ such as DPF and molar extinction coefficients.

\section{Outcome measures, validity and reliability}

When using fNIRS, $\mathrm{HbO} 2$ and $\mathrm{HHb}$ outcomes are generally expressed in units of micro-molar concentration. These measures reflect the change in hemoglobin chromophore concentrations (i.e., neural activity) in the measured cortical regions between the task and baseline condition. Some studies have reported only $\mathrm{HbO} 2$ concentration changes as a measure of direct metabolism of the neural tissues. $\mathrm{HbO} 2$ measures are also more expressive of change due to a higher signal-to-noise ratio than $\mathrm{HHb}[140,141] . \mathrm{HbO} 2$, however, has been shown to be more susceptible to systemic contributions (i.e., increased heart rate) that may not be associated with the task performed $[123,142]$. Thus it is recommended to also report changes in $\mathrm{HHb}$ which have been shown to correlate closely with the BOLD signal [143]. Furthermore, there is evidence that the strength of the correlation between $\mathrm{HbO} 2$ and $\mathrm{HHb}$ is a marker of the amount of artefact affecting the signal [144].

By definition, $\mathrm{HbO} 2$ and $\mathrm{HHb}$ exist in equilibrium, such that an increase in one results in a stoichiometric decrease in the other. But this explanation is only valid if regional blood volume is constant. Much of the available research using fNIRS during gait and posture is on older 
adults $[62,63,66,68,69,71]$ and neurological patients $[59,63,66,68,145]$. These populations often have asymmetrical neural pathologies and vascular disease, which may affect hemodynamics. As such, additional measures have been calculated from $\mathrm{HbO} 2$ and $\mathrm{HHb}$. These include for example, the total hemoglobin ( $\mathrm{HbTotal}=\mathrm{HbO} 2+\mathrm{HHb})$, the tissue oxygenation index which may be expressed as the change in $\mathrm{HbO} 2$ relative to the change in $\mathrm{HHb}[146]$, the ratio of $\mathrm{HbO} 2$ to $\mathrm{HbTotal}[53,147]$, the difference between hemoglobin species $(\mathrm{HbDiff}=\mathrm{HbO} 2-\mathrm{HHb})[31]$ and the regional cortical activation ratio ( $\mathrm{HbO} 2$ measured at a single channel over the ROI divided by average $\mathrm{HbO} 2$ of all channels multiplied by 100) [33]. These measures reflect the systems' ability to utilize (consumption) and replenish (supply) $\mathrm{HbO} 2$ and provide additional insight into task activity and performance. Studies have used different outcome measures to quantify fNIRS data: mean values, median values, peak values, area under the curve, slope, time to peak (see in reviews $[70,104]$ ); their choice generally relate to the distribution of the data and the research question. Regardless of the choice of outcome measure, measures of variability such as standard deviation, standard error, confidence interval, range or interquartile range should always be provided.

\section{Validity and Reliability}

Numerous studies have been conducted to cross-validate fNIRS through comparison with other modalities. Several studies have shown comparable fNIRS signals to functional MRI $[148,149]$ when measured simultaneously (see [150] for a review). Brain activations have also been compared between similar tasks, such as imagined balance/gait tasks in an MRI scanner versus actual balance/gait tasks with fNIRS (see [72] for a review), and stepping movements while supine in an MRI scanner versus upright stepping using fNIRS [151]. While similarities were found within these studies, the inherent posture-related difference between the tasks 
(i.e. supine versus upright) resulted in many differences in regional activation, not necessarily reflective of the task assessed but rather of the method of assessment. In order to further validate fNIRS for balance and gait tasks, studies have used other portable devices such as electroencephalography $[152,153]$ for comparison. However, the properties of hemodynamic response versus electrical physiological response again, are quite different. Thus, cross-validation of fNIRS against other instruments during balance and gait remains a challenge which should be further explored.

Sensitivity and specificity are further important validity components of fNIRS measures. Determination of sensitivity and specificity of fNIRS devices leads to information about the credibility of outcomes [154]. This knowledge may allow assessment of hemispheric asymmetry during locomotion tasks that have, as of yet, not been investigated with fNIRS in relation to physical training interventions [22]. Theories about hemisphere behaviour during locomotion; e.g. the complementary hypothesis [155] and the compensation hypothesis [156, 157], could be tested in ecologically valid scenarios provided fNIRS shows acceptable levels of specificity and sensitivity.

Despite the increasing number of published fNIRS studies assessing posture and gait (e.g. [58, 60-72]), only a few papers reported test-retest reliability. Studies exploring this important attribute with motor tasks (i.e., handgrip tasks in people with and without traumatic brain injury [158]; digit manipulation in healthy people [84]) have reported good to moderate testretest reliability of fNIRS data in the prefrontal and motor cortices. These studies have also shown that both task and signal type influence reliability. $\mathrm{HbO} 2$ signals were more reliable overall, than $\mathrm{HHb}$ signals, while tasks involving larger movements were less reliable. These 


\section{Conclusions and future directions}

fNIRS research in gait and posture is in its relative infancy. This consensus statement represents the current state of knowledge and will require updating as new evidence is produced. We provide a set of guidelines for research but by all means do not intend to 
negate novel fNIRS evidence development. Nonetheless, at the time when research in this area is expanding, it is important to ensure standardization and replication thus, transparency is essential. A number of key components are important for replication of fNIRS research. These include detailing the method of data collection, device specification and signal processing techniques (Table S1).

fNIRS relies on an external placement of recording optodes to guide signal interpretation [80, 160]. An accurate description of the relations between external anatomical landmarks on the scalp and the cortical anatomy beneath is therefore crucial to draw valid conclusions from the measured brain activity with fNIRS [161]. Robust functional inference from the recorded signals can also be facilitated by averaging across channels of ROIs and trials $[61,104,160]$. Different methods have been suggested to determine such ROIs [160, 162]. The choice of ROI and location of the optodes can both impact interpretation of the results.

As a result of certain neurological conditions, the interpretation of brain activation across certain ROls may be problematic. Currently, it is unclear if there are abnormal hemodynamic responses over lesioned areas or peri-lesional areas. Some groups have reported abnormalities in neurovascular coupling post-stroke $[163,164]$ and in near infrared lighttissue interaction in the case of hematomas [165]. This may challenge interpretation as suboptimal neurovascular coupling might be a result of the actual brain pathology (e.g. ischemic regions, arteriosclerosis) or pathological brain function (e.g. neural recruitment or compensation). As one example, we can consider how an asymmetrical brain pathology can impact bilateral activities such as balance and gait. It is therefore strongly recommended to provide explicit and informative definitions for ROls including justification of the number and 
location of channels. In addition, for studies including clinical groups, a description of any brain lesions present and their proximity to fNIRS channels should be provided.

All processing steps and any assumptions made (e.g. the DPF value) should be clearly outlined in reports of fNIRS data. Channel-wise analyses may be impacted by variations in head sizes and shapes between participants. This should be taken into consideration. Methods used for channel localization on the scalp, as well as their spatial registration technique should be detailed. To move the field forward, it is essential to find techniques to account for anatomical anomalies to ensure valid findings. Exploration beyond the single ROI is extremely interesting and includes investigating functional connectomes in a similar way to fMRI [166]. This area is still not developed in the field of fNIRS [167] mainly since this type of approach requires multiple optode locations to cover the whole brain. Recently introduced devices offer whole brain fNIRS coverage, as such, we expect this area will grow and complement the existing neuroimaging literature.

fNIRS data collection methods require repeated trials, which over time, can jeopardize signal quality by reducing signal-to-noise ratio and eventually leading to missing data [89]. Moreover, trials severely contaminated by motion artefacts and/or strong physiological noise are commonly rejected, whether automatically or based on visual inspection [168]. An a priori approach to data removal should be set. The amount of missing data (i.e. number of excluded channels, trials, and/or participants) and how this was accounted for in the analysis should be transparent in the reporting of fNIRS studies. Similarly, the software and specific processing pipelines used should also be described in order to ensure reproducibility of fNIRS findings. Future studies that systematically compare different filter methods are necessary before an 
evidence-based recommendation can be given. Models incorporating multiple physiological confounders may help to better identify the physiological origin of signal changes and help to further elucidate neural function [90]. Table 1 provides a summary of key point recommendations and considerations while Table S1 provides more specific guidance regarding methodological details that should be reported in order to enhance interpretation of research findings.

Inter-individual differences in cognitive, psychological and physical functions are highly significant not only across disease populations but also in normal aging. Among healthy older adults, variables such as gender and stress [169], gait abnormalities [170], levels of fatigue [171] as well as structural brain differences in grey matter volume [27] and white matter integrity [172] have major effects on fNIRS-derived hemodynamic responses. Moreover, improved efficiency in fNIRS-derived activation patterns due to practice in one session [26] was greatly affected by the presence of fear of falls [173]. Hence, due to the inherent heterogeneity in disease populations and healthy older adults the sample size should be carefully considered and resources should be explicitly allocated to maximize the number of participants. Furthermore, detailed characterization of the participants in terms of relevant demographic and clinical variables should be provided. Such information will be critical for replication and test-retest reliability studies as well as for investigations that are specifically designed to evaluate the utility of fNIRS as primary or secondary outcome measure in clinical trials.

Lastly, to advance the field, researchers should consider data sharing through open science repositories. This will allow researchers to compare their data and processing algorithms with 
others directly, instead of indirectly through published reports. Such repositories are becoming increasingly common in the imaging field such as in MRI research (e.g., International Data-sharing Neuroimaging Initiative: INDI from the Consortium for Reliability and Reproducibility (CoRR) [174] and the CBS Neuroimaging Repository [175]) as they can stimulate the development of data processing tools, facilitate reproducibility and collaboration. The added advantage of open science repositories is that it makes research products open to everyone. This in turn accelerates the identification and understanding of the neural underpinnings involved during posture and gait tasks. 
Author contributions: JM and $\mathrm{AM}$ designed the concept of this manuscript, led the collaborative writing and reviewing efforts, and edited the final draft of the manuscript. All authors contributed to the redaction and reviewing of the manuscript. 


\section{REFERENCES}

1. H. Owen-Reece, M. Smith, C.E. Elwell, J.C. Goldstone, Near infrared spectroscopy, Br J Anaesth. 82 (1999) 418-426. 10.1093/bja/82.3.418.

2. T. Csipo, P. Mukli, A. Lipecz, S. Tarantini, D. Bahadli, O. Abdulhussein, et al., Assessment of age-related decline of neurovascular coupling responses by functional near-infrared spectroscopy (fNIRS) in humans, Geroscience. 41 (2019) 495-509. 10.1007/s11357-01900122-x.

3. M. Fabiani, B.A. Gordon, E.L. Maclin, M.A. Pearson, C.R. Brumback-Peltz, K.A. Low, et al., Neurovascular coupling in normal aging: a combined optical, ERP and fMRI study, Neuroimage. 85 Pt 1 (2014) 592-607. 10.1016/j.neuroimage.2013.04.113.

4. J. Steinbrink, A. Villringer, F. Kempf, D. Haux, S. Boden, H. Obrig, Illuminating the BOLD signal: combined fMRI-fNIRS studies, Magn Reson Imaging. 24 (2006) 495-505. 10.1016/j.mri.2005.12.034.

5. H. Karim, B. Schmidt, D. Dart, N. Beluk, T. Huppert, Functional near-infrared spectroscopy (fNIRS) of brain function during active balancing using a video game system, Gait Posture. 35 (2012) 367-372. http://dx.doi.org/10.1016/j.gaitpost.2011.10.007.

6. T. Huppert, B. Schmidt, N. Beluk, J. Furman, P. Sparto, Measurement of brain activation during an upright stepping reaction task using functional near-infrared spectroscopy, Hum Brain Mapp. 34 (2013) 2817-2828. http://dx.doi.org/10.1002/hbm.22106.

7. A.L. Rosso, M. Cenciarini, P.J. Sparto, P.J. Loughlin, J.M. Furman, T.J. Huppert, Neuroimaging of an attention demanding dual-task during dynamic postural control, Gait Posture. 57 (2017) 193-198. http://dx.doi.org/10.1016/j.gaitpost.2017.06.013.

8. S. Basso Moro, S. Bisconti, M. Muthalib, M. Spezialetti, S. Cutini, M. Ferrari, et al., A semiimmersive virtual reality incremental swing balance task activates prefrontal cortex: A functional near-infrared spectroscopy study, Neurolmage. 85 (2014) 451-460. http://dx.doi.org/10.1016/j.neuroimage.2013.05.031. 
9. A.B. Rosen, J.M. Yentes, M.L. McGrath, A.C. Maerlender, S.A. Myers, M. Mukherjee, Alterations in Cortical Activation Among Individuals With Chronic Ankle Instability During Single-Limb Postural Control, J Athl Train. 54 (2019) 718-726. http://dx.doi.org/10.4085/10626050-448-17.

10. W.P. Teo, A.M. Goodwill, A.M. Hendy, M. Muthalib, H. Macpherson, Sensory manipulation results in increased dorsolateral prefrontal cortex activation during static postural balance in sedentary older adults: An fNIRS study, Brain Behav. 8 (2018) http://dx.doi.org/10.1002/brb3.1109.

11. A.C. de Lima-Pardini, G.A. Zimeo Morais, J.B. Balardin, D.B. Coelho, N.M. Azzi, L.A. Teixeira, et al., Measuring cortical motor hemodynamics during assisted stepping - An fNIRS feasibility study of using a walker, Gait Posture. $56 \quad$ (2017) 112-118. http://dx.doi.org/10.1016/j.gaitpost.2017.05.018.

12. A. Mirelman, I. Maidan, H. Bernad-Elazari, F. Nieuwhof, M. Reelick, N. Giladi, et al., Increased frontal brain activation during walking while dual tasking: An fNIRS study in healthy young adults, J Neuroeng Rehabil. 11 (2014) http://dx.doi.org/10.1186/1743-0003-11-85.

13. R. Holtzer, J.R. Mahoney, M. Izzetoglu, C. Wang, S. England, J. Verghese, Online fronto-cortical control of simple and attention-demanding locomotion in humans, Neurolmage. 112 (2015) 152-159. http://dx.doi.org/10.1016/j.neuroimage.2015.03.002.

14. M. Chen, S. Pillemer, S. England, M. Izzetoglu, J.R. Mahoney, R. Holtzer, Neural correlates of obstacle negotiation in older adults: An fNIRS study, Gait Posture. 58 (2017) 130-135. http://dx.doi.org/10.1016/j.gaitpost.2017.07.043.

15. A. Mirelman, I. Maidan, H. Bernad-Elazari, S. Shustack, N. Giladi, J.M. Hausdorff, Effects of aging on prefrontal brain activation during challenging walking conditions, Brain Cogn. 115 (2017) 41-46. http://dx.doi.org/10.1016/j.bandc.2017.04.002. 
16. I. Maidan, S. Shustak, T. Sharon, H. Bernad-Elazari, N. Geffen, N. Giladi, et al., Prefrontal cortex activation during obstacle negotiation: What's the effect size and timing?, Brain Cogn. 122 (2018) 45-51. http://dx.doi.org/10.1016/j.bandc.2018.02.006.

17. K.A. Hawkins, E.J. Fox, J.J. Daly, D.K. Rose, E.A. Christou, T.E. McGuirk, et al., Prefrontal overactivation during walking in people with mobility deficits: Interpretation and functional implications, Hum Mov Sci. 59 (2018) 46-55. http://dx.doi.org/10.1016/j.humov.2018.03.010.

18. M.J. Kurz, T.W. Wilson, D.J. Arpin, Stride-time variability and sensorimotor cortical activation $\begin{array}{llll}\text { during } \quad \text { walking, } & \text { Neurolmage. } & 59 & \text { (2012) }\end{array}$ http://dx.doi.org/10.1016/j.neuroimage.2011.08.084.

19. R. Beurskens, I. Helmich, R. Rein, O. Bock, Age-related changes in prefrontal activity during walking in dual-task situations: A fNIRS study, Int J Psychophysiol. 92 (2014) 122-128. http://dx.doi.org/10.1016/j.ijpsycho.2014.03.005.

20. K.L.M. Koenraadt, E.G.J. Roelofsen, J. Duysens, N.L.W. Keijsers, Cortical control of normal gait and precision stepping: An fNIRS study, Neurolmage. 85 (2014) 415-422. http://dx.doi.org/10.1016/j.neuroimage.2013.04.070.

21. D. Meester, E. Al-Yahya, H. Dawes, P. Martin-Fagg, C. Pinon, Associations between prefrontal cortex activation and H-reflex modulation during dual task gait, Front Hum Neurosci. 8 (2014) http://dx.doi.org/10.3389/fnhum.2014.00078.

22. P. Eggenberger, M. Wolf, M. Schumann, E.D. de Bruin, Exergame and balance training modulate prefrontal brain activity during walking and enhance executive function in older adults, Front Aging Neurosci. 8 (2016) http://dx.doi.org/10.3389/fnagi.2016.00066.

23. S.A. Fraser, O. Dupuy, P. Pouliot, F. Lesage, L. Bherer, Comparable cerebral oxygenation patterns in younger and older adults during dual-task walking with increasing load, Front Aging Neurosci. 8 (2016) http://dx.doi.org/10.3389/fnagi.2016.00240. 
24. T. Harada, I. Miyai, M. Suzuki, K. Kubota, Gait capacity affects cortical activation patterns related to speed control in the elderly, Exp Brain Res. 193 (2009) 445-454. http://dx.doi.org/10.1007/s00221-008-1643-y.

25. C.J. George, J. Verghese, M. Izzetoglu, C. Wang, R. Holtzer, The effect of polypharmacy on prefrontal cortex activation during single and dual task walking in community dwelling older adults, Pharmacol Res. 139 (2019) 113-119. http://dx.doi.org/10.1016/j.phrs.2018.11.007.

26. R. Holtzer, M. Izzetoglu, M. Chen, C. Wang, Distinct fNIRS-Derived HbO2 Trajectories During the Course and Over Repeated Walking Trials Under Single- and Dual-Task Conditions: Implications for Within Session Learning and Prefrontal Cortex Efficiency in Older Adults, J Gerontol A Biol Sci Med Sci. 74 (2019) 1076-1083. http://dx.doi.org/10.1093/gerona/gly181.

27. M.E. Wagshul, M. Lucas, K. Ye, M. Izzetoglu, R. Holtzer, Multi-modal neuroimaging of dualtask walking: Structural MRI and fNIRS analysis reveals prefrontal grey matter volume moderation of brain activation in older adults, Neurolmage. 189 (2019) 745-754. http://dx.doi.org/10.1016/j.neuroimage.2019.01.045.

28. S. Stuart, L. Alcock, L. Rochester, R. Vitorio, A. Pantall, Monitoring multiple cortical regions during walking in young and older adults: Dual-task response and comparison challenges, Int. J. Psychophysiol. 135 (2019) 63-72. http://dx.doi.org/10.1016/j.ijpsycho.2018.11.006.

29. F.G. Metzger, A.C. Ehlis, F.B. Haeussinger, P. Schneeweiss, J. Hudak, A.J. Fallgatter, et al., Functional brain imaging of walking while talking - An fNIRS study, Neuroscience. 343 (2017) 85-93. http://dx.doi.org/10.1016/j.neuroscience.2016.11.032.

30. R. Holtzer, J.R. Mahoney, M. Izzetoglu, K. Izzetoglu, B. Onaral, J. Verghese, fNIRS study of walking and walking while talking in young and old individuals, J Gerontol A Biol Sci Med Sci. 66 (2011) 879-887. 10.1093/gerona/g|r068.

31. C.F. Lu, Y.C. Liu, Y.R. Yang, Y.T. Wu, R.Y. Wang, Maintaining gait performance by cortical activation during dual-task interference: A functional near-infrared spectroscopy study, PLoS One. 10 (2015) http://dx.doi.org/10.1371/journal.pone.0129390. 
32. M. Suzuki, I. Miyai, T. Ono, K. Kubota, Activities in the frontal cortex and gait performance are modulated by preparation. An fNIRS study, Neurolmage. 39 (2008) 600-607. http://dx.doi.org/10.1016/j.neuroimage.2007.08.044.

33. M. Suzuki, I. Miyai, T. Ono, I. Oda, I. Konishi, T. Kochiyama, et al., Prefrontal and premotor cortices are involved in adapting walking and running speed on the treadmill: an optical imaging study, Neuroimage. 23 (2004) 1020-1026. 10.1016/j.neuroimage.2004.07.002.

34. V. Belluscio, S. Stuart, E. Bergamini, G. Vannozzi, M. Mancini, The Association between Prefrontal Cortex Activity and Turning Behavior in People with and without Freezing of Gait, Neuroscience. 416 (2019) 168-176. http://dx.doi.org/10.1016/j.neuroscience.2019.07.024.

35. I. Maidan, H. Bernad-Elazari, E. Gazit, N. Giladi, J.M. Hausdorff, A. Mirelman, Changes in oxygenated hemoglobin link freezing of gait to frontal activation in patients with Parkinson disease: an fNIRS study of transient motor-cognitive failures, J Neurol. 31 (2015) http://dx.doi.org/10.1007/s00415-015-7650-6.

36. I. Maidan, H. Bernad-Elazari, N. Giladi, J.M. Hausdorff, A. Mirelman, When is Higher Level Cognitive Control Needed for Locomotor Tasks Among Patients with Parkinson's Disease?, Brain Topogr. 30 (2017) 531-538. http://dx.doi.org/10.1007/s10548-017-0564-0.

37. I. Maidan, F. Nieuwhof, H. Bernad-Elazari, B.R. Bloem, N. Giladi, J.M. Hausdorff, et al., Evidence for Differential Effects of 2 Forms of Exercise on Prefrontal Plasticity During Walking in Parkinson's Disease, Neurorehabil Neural Repair. 32 (2018) 200-208. http://dx.doi.org/10.1177/1545968318763750.

38. I. Maidan, F. Nieuwhof, H. Bernad-Elazari, M.F. Reelick, B.R. Bloem, N. Giladi, et al., The Role of the Frontal Lobe in Complex Walking among Patients with Parkinson's Disease and Healthy Older Adults: An fNIRS Study, Neurorehabil Neural Repair. 30 (2016) 963-971. http://dx.doi.org/10.1177/1545968316650426. 
39. S. Stuart, V. Belluscio, J.F. Quinn, M. Mancini, Pre-frontal cortical activity during walking and turning is reliable and differentiates across young, older adults and people with Parkinson's disease, Front Neurol. 10 (2019) http://dx.doi.org/10.3389/fneur.2019.00536.

40. S. Stuart, M. Mancini, Prefrontal Cortical Activation With Open and Closed-Loop Tactile Cueing When Walking and Turning in Parkinson Disease: A Pilot Study, J Neurolc Phys Ther. 14 (2019) http://dx.doi.org/10.1097/NPT.0000000000000286.

41. P.C. Thumm, I. Maidan, M. Brozgol, S. Shustak, E. Gazit, S. Shema Shiratzki, et al., Treadmill walking reduces pre-frontal activation in patients with Parkinson's disease, Gait Posture. 62 (2018) 384-387. http://dx.doi.org/10.1016/j.gaitpost.2018.03.041.

42. S.A. Chatterjee, E.J. Fox, J.J. Daly, D.K. Rose, S.S. Wu, E.A. Christou, et al., Interpreting prefrontal recruitment during walking after stroke: Influence of individual differences in mobility and cognitive function, Front Hum Neurosci. 13 (2019) http://dx.doi.org/10.3389/fnhum.2019.00194.

43. H. Fujimoto, M. Mihara, N. Hattori, M. Hatakenaka, T. Kawano, H. Yagura, et al., Cortical changes underlying balance recovery in patients with hemiplegic stroke, Neurolmage. 85 (2014) 547-554. http://dx.doi.org/10.1016/j.neuroimage.2013.05.014.

44. E. Hermand, B. Tapie, O. Dupuy, S. Fraser, M. Compagnat, J.Y. Salle, et al., Prefrontal cortex activation during dual task with increasing cognitive load in subacute stroke patients: A pilot study, Front Aging Neurosci. 10 (2019) http://dx.doi.org/10.3389/fnagi.2019.00160.

45. Y.C. Liu, Y.R. Yang, Y.A. Tsai, R.Y. Wang, C.F. Lu, Brain Activation and Gait Alteration during Cognitive and Motor Dual Task Walking in Stroke-A Functional Near-Infrared Spectroscopy Study, IEEE Trans Neural Syst Rehabil Eng. $26 \quad$ (2018) 2416-2423. http://dx.doi.org/10.1109/TNSRE.2018.2878045.

46. M. Mihara, I. Miyai, M. Hatakenaka, K. Kubota, S. Sakoda, Sustained prefrontal activation during ataxic gait: A compensatory mechanism for ataxic stroke?, Neurolmage. 37 (2007) 1338-1345. http://dx.doi.org/10.1016/j.neuroimage.2007.06.014. 
47. M. Mihara, I. Miyai, N. Hattori, M. Hatakenaka, H. Yagura, et al., Cortical control of postural balance in patients with hemiplegic stroke, NeuroReport. 18 (2012) http://dx.doi.org/10.1097/WNR.0b013e328351757b.

48. M. Rea, M. Rana, N. Lugato, P. Terekhin, L. Gizzi, D. Brotz, et al., Lower limb movement preparation in chronic stroke: A pilot study toward an fNIRS-BCl for gait rehabilitation, $\begin{array}{lllll}\text { Neurorehabil } & \text { Neural } & \text { Repair. } & 28 & \text { (2014) }\end{array}$ http://dx.doi.org/10.1177/1545968313520410.

49. G. Chaparro, J.M. Balto, B.M. Sandroff, R. Holtzer, M. Izzetoglu, R.W. Motl, et al., Frontal brain activation changes due to dual-tasking under partial body weight support conditions in older adults with multiple sclerosis, J Neuroeng Rehabil. 14 (2017) http://dx.doi.org/10.1186/s12984-017-0280-8.

50. M.E. Hernandez, R. Holtzer, G. Chaparro, K. Jean, J.M. Balto, B.M. Sandroff, et al., Brain activation changes during locomotion in middle-aged to older adults with multiple sclerosis, J Neurol Sci. 370 (2016) 277-283. http://dx.doi.org/10.1016/j.jns.2016.10.002.

51. M.E. Hernandez, E. O'Donnell, G. Chaparro, R. Holtzer, M. Izzetoglu, B.M. Sandroff, et al., Brain activation changes during balance- And attention-demanding tasks in middle- And older-aged adults with multiple sclerosis, Motor Control. 23 (2019) 498-517. http://dx.doi.org/10.1123/mc.2018-0044.

52. S. Saleh, B.M. Sandroff, T. Vitiello, O. Owoeye, A. Hoxha, P. Hake, et al., The role of premotor areas in dual tasking in healthy controls and persons with multiple sclerosis: An fNIRS imaging study, Front Behav Neurosci. 12 (2018) http://dx.doi.org/10.3389/fnbeh.2018.00296.

53. D.J. Clark, E.A. Christou, S.A. Ring, J.B. Williamson, L. Doty, Enhanced somatosensory feedback reduces prefrontal cortical activity during walking in older adults, J Gerontol A Biol Sci Med Sci. 69 (2014) 1422-1428. http://dx.doi.org/10.1093/gerona/glu125. 
54. R. Holtzer, B.C. Rakitin, J. Steffener, J. Flynn, A. Kumar, Y. Stern, Age effects on load-dependent brain activations in working memory for novel material, Brain Res. 1249 (2009) 148-161. 10.1016/j.brainres.2008.10.009.

55. Y. Stern, What is cognitive reserve? Theory and research application of the reserve concept, J Int Neuropsychol Soc. 8 (2002) 448-460.

56. Y. Stern, Cognitive reserve, Neuropsychologia. 47 (2009) 2015-2028. 10.1016/j.neuropsychologia.2009.03.004.

57. S.M. Daselaar, V. Iyengar, S.W. Davis, K. Eklund, S.M. Hayes, R.E. Cabeza, Less wiring, more firing: low-performing older adults compensate for impaired white matter with greater neural activity, Cereb Cortex. 25 (2015) 983-990. 10.1093/cercor/bht289.

58. A. Berger, F. Horst, S. Muller, F. Steinberg, M. Doppelmayr, Current state and future prospects of EEG and fNIRS in robot-assisted gait rehabilitation: A brief review, Front Hum Neurosci. 13 (2019) http://dx.doi.org/10.3389/fnhum.2019.00172.

59. V. Gramigna, G. Pellegrino, A. Cerasa, S. Cutini, R. Vasta, G. Olivadese, et al., Near-Infrared Spectroscopy in Gait Disorders: Is It Time to Begin?, Neurorehabil Neural Repair. 31 (2017) 402-412. http://dx.doi.org/10.1177/1545968317693304.

60. F. Herold, P. Wiegel, D. Hamacher, L. Schega, Brain activity during walking: A systematic $\begin{array}{lllll}\text { review, Neurosci } & \text { Biobehav } & \text { 310-327. }\end{array}$ http://dx.doi.org/10.1016/j.neubiorev.2015.08.002.

61. F. Herold, P. Wiegel, F. Scholkmann, A. Thiers, D. Hamacher, L. Schega, Functional nearinfrared spectroscopy in movement science: A systematic review on cortical activity in $\begin{array}{lllll}\text { postural and walking tasks, Neurophotonics. } & 4 & \text { (2017) }\end{array}$ http://dx.doi.org/10.1117/1.NPh.4.4.041403.

62. R. Holtzer, N. Epstein, J.R. Mahoney, M. Izzetoglu, H.M. Blumen, Neuroimaging of mobility in aging: a targeted review, J Gerontol A Biol Sci Med Sci. 69 (2014) 1375-1388. http://dx.doi.org/10.1093/gerona/glu052. 
63. M. Kahya, S. Moon, M. Ranchet, R.R. Vukas, K.E. Lyons, R. Pahwa, et al., Brain activity during dual task gait and balance in aging and age-related neurodegenerative conditions: A systematic review, Exp Gerontol. 128 (2019) 110756. 10.1016/j.exger.2019.110756.

64. D.R. Leff, F. Orihuela-Espina, C.E. Elwell, T. Athanasiou, D.T. Delpy, A.W. Darzi, et al., Assessment of the cerebral cortex during motor task behaviours in adults: A systematic review of functional near infrared spectroscopy (fNIRS) studies, Neurolmage. 54 (2011) 2922-2936. http://dx.doi.org/10.1016/j.neuroimage.2010.10.058.

65. M. Mihara, I. Miyai, Review of functional near-infrared spectroscopy in neurorehabilitation, Neurophotonics. 3 (2016) http://dx.doi.org/10.1117/1.NPh.3.3.031414.

66. P.H.S. Pelicioni, M. Tijsma, S.R. Lord, J. Menant, Prefrontal cortical activation measured by fNIRS during walking: effects of age, disease and secondary task, PeerJ. 7 (2019) e6833. 10.7717/peerj.6833.

67. V. Quaresima, M. Ferrari, A Mini-Review on Functional Near-Infrared Spectroscopy (fNIRS): Where Do We Stand, and Where Should We Go?, Photonics. 6 (2019)

68. S. Stuart, R. Vitorio, R. Morris, D.N. Martini, P.C. Fino, M. Mancini, Cortical activity during walking and balance tasks in older adults and in people with Parkinson's disease: A structured review, Maturitas. 113 (2018) 53-72. http://dx.doi.org/10.1016/j.maturitas.2018.04.011.

69. C. Udina, S. Avtzi, T. Durduran, R. Holtzer, A.L. Rosso, C. Castellano-Tejedor, et al., Functional Near-Infrared Spectroscopy to Study Cerebral Hemodynamics in Older Adults During Cognitive and Motor Tasks: A Review, Front Aging Neurosci. 11 (2019) 367. 10.3389/fnagi.2019.00367.

70. R. Vitorio, S. Stuart, L. Rochester, L. Alcock, A. Pantall, fNIRS response during walking - Artefact or cortical activity? A systematic review, Neurosci Biobehav Rev. 83 (2017) 160-172. http://dx.doi.org/10.1016/j.neubiorev.2017.10.002.

71. J. Wilson, L. Allcock, R. Mc Ardle, J.P. Taylor, L. Rochester, The neural correlates of discrete gait characteristics in ageing: A structured review, Neurosci Biobehav Rev. 100 (2019) 344369. http://dx.doi.org/10.1016/j.neubiorev.2018.12.017. 
72. D. Hamacher, F. Herold, P. Wiegel, D. Hamacher, L. Schega, Brain activity during walking: A systematic review, Neurosci Biobehav Rev. $57 \quad$ (2015) 310-327. 10.1016/j.neubiorev.2015.08.002.

73. F. Scholkmann, S. Kleiser, A.J. Metz, R. Zimmermann, J. Mata Pavia, U. Wolf, et al., A review on continuous wave functional near-infrared spectroscopy and imaging instrumentation and methodology, Neuroimage. 85 Pt 1 (2014a) 6-27. 10.1016/j.neuroimage.2013.05.004.

74. L. Wang, H. Ayaz, M. Izzetoglu, B. Onaral, Evaluation of light detector surface area for functional Near Infrared Spectroscopy, Comput Biol Med. 89 (2017) 68-75. 10.1016/j.compbiomed.2017.07.019.

75. X. Cui, S. Bray, A.L. Reiss, Speeded near infrared spectroscopy (NIRS) response detection, PLoS One. 5 (2010) e15474. 10.1371/journal.pone.0015474.

76. M.L. Schroeter, S. Zysset, D.Y. von Cramon, Shortening intertrial intervals in event-related cognitive studies with near-infrared spectroscopy, Neuroimage. 22 (2004) 341-346. 10.1016/j.neuroimage.2003.12.041.

77. I. Tachtsidis, C.E. Elwell, T.S. Leung, C.W. Lee, M. Smith, D.T. Delpy, Investigation of cerebral haemodynamics by near-infrared spectroscopy in young healthy volunteers reveals posturedependent spontaneous oscillations, Physiol Meas. 25 (2004) 437-445. 10.1088/0967$3334 / 25 / 2 / 003$.

78. G.H. Klem, H.O. Luders, H.H. Jasper, C. Elger, The ten-twenty electrode system of the International Federation. The International Federation of Clinical Neurophysiology, Electroencephalogr Clin Neurophysiol Suppl. 52 (1999) 3-6.

79. V. Jurcak, D. Tsuzuki, I. Dan, $10 / 20,10 / 10$, and $10 / 5$ systems revisited: their validity as relative head-surface-based positioning systems, Neuroimage. $34 \quad$ (2007) 1600-1611. 10.1016/j.neuroimage.2006.09.024. 
80. G.A. Zimeo Morais, J.B. Balardin, J.R. Sato, fNIRS Optodes' Location Decider (fOLD): a toolbox for probe arrangement guided by brain regions-of-interest, Sci Rep. 8 (2018) 3341. 10.1038/s41598-018-21716-z.

81. A.K. Singh, M. Okamoto, H. Dan, V. Jurcak, I. Dan, Spatial registration of multichannel multisubject fNIRS data to MNI space without MRI, Neuroimage. 27 (2005) 842-851. 10.1016/j.neuroimage.2005.05.019.

82. A. Alexander-Bloch, J.N. Giedd, E. Bullmore, Imaging structural co-variance between human brain regions, Nat Rev Neurosci. 14 (2013) 322-336. 10.1038/nrn3465.

83. J. Ashburner, J.G. Csernansky, C. Davatzikos, N.C. Fox, G.B. Frisoni, P.M. Thompson, Computerassisted imaging to assess brain structure in healthy and diseased brains, Lancet Neurol. 2 (2003) 79-88. 10.1016/s1474-4422(03)00304-1.

84. S. Dravida, J.A. Noah, X. Zhang, J. Hirsch, Comparison of oxyhemoglobin and deoxyhemoglobin signal reliability with and without global mean removal for digit manipulation motor tasks, Neurophotonics. 5 (2018) 011006. 10.1117/1.NPh.5.1.011006.

85. J.B. Balardin, G.A. Zimeo Morais, R.A. Furucho, L.R. Trambaiolli, J.R. Sato, Impact of communicative head movements on the quality of functional near-infrared spectroscopy signals: negligible effects for affirmative and negative gestures and consistent artifacts related to raising eyebrows, J Biomed Opt. 22 (2017) 46010. 10.1117/1.Jbo.22.4.046010.

86. G.A. Zimeo Morais, F. Scholkmann, J.B. Balardin, R.A. Furucho, R.C.V. de Paula, C.E. Biazoli, Jr., et al., Non-neuronal evoked and spontaneous hemodynamic changes in the anterior temporal region of the human head may lead to misinterpretations of functional near-infrared spectroscopy signals, Neurophotonics. 5 (2018) 011002. 10.1117/1.NPh.5.1.011002.

87. P. Pinti, C. Aichelburg, S. Gilbert, A. Hamilton, J. Hirsch, P. Burgess, et al., A Review on the Use of Wearable Functional Near-Infrared Spectroscopy in Naturalistic Environments, Jpn Psychol Res. 60 (2018) 347-373. 10.1111/jpr.12206. 
88. L. Pollonini, H. Bortfeld, J.S. Oghalai, PHOEBE: a method for real time mapping of optodesscalp coupling in functional near-infrared spectroscopy, Biomed Opt Express. 7 (2016) 51045119. 10.1364/boe.7.005104.

89. F. Orihuela-Espina, D.R. Leff, D.R. James, A.W. Darzi, G.Z. Yang, Quality control and assurance in functional near infrared spectroscopy (fNIRS) experimentation, Phys Med Biol. 55 (2010) 3701-3724. 10.1088/0031-9155/55/13/009.

90. I. Tachtsidis, F. Scholkmann, False positives and false negatives in functional near-infrared spectroscopy: issues, challenges, and the way forward, Neurophotonics. 3 (2016) 030401. 10.1117/1.NPh.3.3.030401.

91. D. Tomasi, E.C. Caparelli, L. Chang, T. Ernst, fMRI-acoustic noise alters brain activation during working memory tasks, Neuroimage. 27 (2005) 377-386. 10.1016/j.neuroimage.2005.04.010.

92. J.M. Baker, D. Rojas-Valverde, R. Gutierrez, M. Winkler, S. Fuhrimann, B. Eskenazi, et al., Portable Functional Neuroimaging as an Environmental Epidemiology Tool: A How-To Guide for the Use of fNIRS in Field Studies, Environ Health Perspect. 125 (2017) 094502. 10.1289/ehp2049.

93. A.J. Metz, S.D. Klein, F. Scholkmann, U. Wolf, Continuous coloured light altered human brain haemodynamics and oxygenation assessed by systemic physiology augmented functional near-infrared spectroscopy, Sci Rep. 7 (2017) 10027. 10.1038/s41598-017-09970-z.

94. F. Scholkmann, T. Hafner, A.J. Metz, M. Wolf, U. Wolf, Effect of short-term colored-light exposure on cerebral hemodynamics and oxygenation, and systemic physiological activity, Neurophotonics. 4 (2017) 045005. 10.1117/1.NPh.4.4.045005.

95. K. Uludag, J. Steinbrink, A. Villringer, H. Obrig, Separability and cross talk: optimizing dual wavelength combinations for near-infrared spectroscopy of the adult head, Neuroimage. 22 (2004) 583-589. 10.1016/j.neuroimage.2004.02.023. 
96. R.K. Almajidy, K. Mankodiya, M. Abtahi, U.G. Hofmann, A Newcomer's Guide to Functional Near Infrared Spectroscopy Experiments, IEEE Rev Biomed Eng. 13 (2020) 292-308. 10.1109/rbme.2019.2944351.

97. M. Schecklmann, A. Mann, B. Langguth, A.C. Ehlis, A.J. Fallgatter, F.B. Haeussinger, The Temporal Muscle of the Head Can Cause Artifacts in Optical Imaging Studies with Functional Near-Infrared Spectroscopy, Front Hum Neurosci. 11 (2017) 456. 10.3389/fnhum.2017.00456.

98. F. Scholkmann, A.J. Metz, M. Wolf, Measuring tissue hemodynamics and oxygenation by continuous-wave functional near-infrared spectroscopy--how robust are the different calculation methods against movement artifacts?, Physiol Meas. 35 (2014b) 717-734. 10.1088/0967-3334/35/4/717.

99. X. Cui, J.M. Baker, N. Liu, A.L. Reiss, Sensitivity of fNIRS measurement to head motion: an applied use of smartphones in the lab, J Neurosci Methods. 245 (2015) 37-43. 10.1016/j.jneumeth.2015.02.006.

100. A. Metz, M. Wolf, P. Achermann, F. Scholkmann, A New Approach for Automatic Removal of Movement Artifacts in Near-Infrared Spectroscopy Time Series by Means of Acceleration Data, Algorithms. 8 (2015) 1052-1075. doi: 10.3390/a8041052.

101. J. Virtanen, T. Noponen, K. Kotilahti, J. Virtanen, R.J. Ilmoniemi, Accelerometer-based method for correcting signal baseline changes caused by motion artifacts in medical near-infrared spectroscopy, J Biomed Opt. 16 (2011) 087005. 10.1117/1.3606576.

102. L. Gagnon, R.J. Cooper, M.A. Yucel, K.L. Perdue, D.N. Greve, D.A. Boas, Short separation channel location impacts the performance of short channel regression in NIRS, Neuroimage. 59 (2012) 2518-2528. 10.1016/j.neuroimage.2011.08.095.

103. T. Sato, I. Nambu, K. Takeda, T. Aihara, O. Yamashita, Y. Isogaya, et al., Reduction of global interference of scalp-hemodynamics in functional near-infrared spectroscopy using short distance probes, Neuroimage. 141 (2016) 120-132. 10.1016/j.neuroimage.2016.06.054. 
104. F. Herold, P. Wiegel, F. Scholkmann, N.G. Muller, Applications of Functional Near-Infrared Spectroscopy (fNIRS) Neuroimaging in Exercise - Cognition Science: A Systematic, Methodology-Focused Review, J Clin Med. 7 (2018) 10.3390/jcm7120466.

105. A. Janani, M. Sasikala, Investigation of different approaches for noise reduction in functional near-infrared spectroscopy signals for brain-computer interface applications., Neural Comput \& Applic. 4 (2017) 10.1007/s00521-017-2961-4.

106. R.J. Cooper, J. Selb, L. Gagnon, D. Phillip, H.W. Schytz, H.K. Iversen, et al., A systematic comparison of motion artifact correction techniques for functional near-infrared spectroscopy, Front Neurosci. 6 (2012) 147. 10.3389/fnins.2012.00147.

107. H.F. Behrendt, C. Firk, C.A. Nelson, 3rd, K.L. Perdue, Motion correction for infant functional near-infrared spectroscopy with an application to live interaction data, Neurophotonics. 5 (2018) 015004. 10.1117/1.NPh.5.1.015004.

108. S. Brigadoi, L. Ceccherini, S. Cutini, F. Scarpa, P. Scatturin, J. Selb, et al., Motion artifacts in functional near-infrared spectroscopy: a comparison of motion correction techniques applied to real cognitive data, Neuroimage. 85 Pt 1 (2014) 181-191. 10.1016/j.neuroimage.2013.04.082.

109. R. Di Lorenzo, L. Pirazzoli, A. Blasi, C. Bulgarelli, Y. Hakuno, Y. Minagawa, et al., Recommendations for motion correction of infant fNIRS data applicable to multiple data sets and acquisition systems, Neuroimage. $200 \quad$ (2019) 511-527. 10.1016/j.neuroimage.2019.06.056.

110. C. Piazza, A. Bacchetta, A. Crippa, M. Mauri, S. Grazioli, G. Reni, et al. Preprocessing Pipeline for fNIRS Data in Children, in: J. Henriques, N. Neves, P. de Carvalho, (Eds), XV Mediterranean Conference on Medical and Biological Engineering and Computing - MEDICON 2019. MEDICON 2019. IFMBE Proceedings, Springer, Cham, 2020, vol 76.

111. S. Jahani, S.K. Setarehdan, D.A. Boas, M.A. Yucel, Motion artifact detection and correction in functional near-infrared spectroscopy: a new hybrid method based on spline interpolation 
method and Savitzky-Golay filtering, Neurophotonics. $5 \quad$ (2018) 015003. 10.1117/1.NPh.5.1.015003.

112. A. Chaddad, Brain Function Diagnosis Enhanced Using Denoised fNIRS Raw Signals, JBiSE. 7 (2014) 218-227. 10.4236/jbise.2014.74025.

113. M.A. Kamran, M.M. Mannan, M.Y. Jeong, Cortical Signal Analysis and Advances in Functional Near-Infrared Spectroscopy Signal: A Review, Front Hum Neurosci. 10 (2016) 261. 10.3389/fnhum.2016.00261.

114. E. Kirilina, N. Yu, A. Jelzow, H. Wabnitz, A.M. Jacobs, I. Tachtsidis, Identifying and quantifying main components of physiological noise in functional near infrared spectroscopy on the prefrontal cortex, Front Hum Neurosci. 7 (2013) 864. 10.3389/fnhum.2013.00864.

115. F. Scholkmann, S. Spichtig, T. Muehlemann, M. Wolf, How to detect and reduce movement artifacts in near-infrared imaging using moving standard deviation and spline interpolation, Physiol Meas. 31 (2010) 649-662. 10.1088/0967-3334/31/5/004.

116. T.J. Huppert, S.G. Diamond, M.A. Franceschini, D.A. Boas, HomER: a review of time-series analysis methods for near-infrared spectroscopy of the brain, Appl Opt. 48 (2009) D280-298. $10.1364 /$ ao. $48.00 \mathrm{~d} 280$.

117. P. Pinti, F. Scholkmann, A. Hamilton, P. Burgess, I. Tachtsidis, Current Status and Issues Regarding Pre-processing of fNIRS Neuroimaging Data: An Investigation of Diverse Signal Filtering Methods Within a General Linear Model Framework, Front Hum Neurosci. 12 (2018) 505. 10.3389/fnhum.2018.00505.

118. M.A. Yucel, J. Selb, C.M. Aasted, P.Y. Lin, D. Borsook, L. Becerra, et al., Mayer waves reduce the accuracy of estimated hemodynamic response functions in functional near-infrared spectroscopy, Biomed Opt Express. 7 (2016) 3078-3088. 10.1364/boe.7.003078.

119. A. Savitzky, M.J.E. Golay, Smoothing and Differentiation of Data by Simplified Least Squares Procedures, Anal. Chem. 36 (1964) 1627-1639. 10.1021/ac60214a047. 
120. M.D. Pfeifer, F. Scholkmann, R. Labruyere, Signal Processing in Functional Near-Infrared Spectroscopy (fNIRS): Methodological Differences Lead to Different Statistical Results, Front Hum Neurosci. 11 (2017) 641. 10.3389/fnhum.2017.00641.

121. A. Vrana, M.L. Meier, S. Hotz-Boendermaker, B.K. Humphreys, F. Scholkmann, Cortical Sensorimotor Processing of Painful Pressure in Patients with Chronic Lower Back Pain-An Optical Neuroimaging Study using fNIRS, Front Hum Neurosci. 10 (2016) 578. 10.3389/fnhum.2016.00578.

122. A. Vrana, M.L. Meier, S. Hotz-Boendermaker, B.K. Humphreys, F. Scholkmann, Different mechanosensory stimulations of the lower back elicit specific changes in hemodynamics and oxygenation in cortical sensorimotor areas-A fNIRS study, Brain Behav. 6 (2016) e00575. 10.1002/brb3.575.

123. E. Kirilina, A. Jelzow, A. Heine, M. Niessing, H. Wabnitz, R. Bruhl, et al., The physiological origin of task-evoked systemic artefacts in functional near infrared spectroscopy, Neuroimage. 61 (2012) 70-81. 10.1016/j.neuroimage.2012.02.074.

124. T. Takahashi, Y. Takikawa, R. Kawagoe, S. Shibuya, T. Iwano, S. Kitazawa, Influence of skin blood flow on near-infrared spectroscopy signals measured on the forehead during a verbal fluency task, Neuroimage. 57 (2011) 991-1002. 10.1016/j.neuroimage.2011.05.012.

125. L. Duan, Z. Zhao, Y. Lin, X. Wu, Y. Luo, P. Xu, Wavelet-based method for removing global physiological noise in functional near-infrared spectroscopy, Biomed Opt Express. 9 (2018) 3805-3820. 10.1364/boe.9.003805.

126. M.A. Yucel, J.J. Selb, T.J. Huppert, M.A. Franceschini, D.A. Boas, Functional Near Infrared Spectroscopy: Enabling Routine Functional Brain Imaging, Curr Opin Biomed Eng. 4 (2017) 7886. 10.1016/j.cobme.2017.09.011.

127. H. Santosa, A. Aarabi, S.B. Perlman, T.J. Huppert, Characterization and correction of the falsediscovery rates in resting state connectivity using functional near-infrared spectroscopy, J Biomed Opt. 22 (2017) 55002. 10.1117/1.Jbo.22.5.055002. 
128. M. Caldwell, F. Scholkmann, U. Wolf, M. Wolf, C. Elwell, I. Tachtsidis, Modelling confounding effects from extracerebral contamination and systemic factors on functional near-infrared spectroscopy, Neuroimage. 143 (2016) 91-105. 10.1016/j.neuroimage.2016.08.058.

129. M. Cope, D.T. Delpy, E.O.R. Reynolds, S. Wray, J. Wyatt, P. van der Zee, Methods of Quantitating Cerebral Near Infrared Spectroscopy Data, in: M. Mochizuki, et al. (Eds.), Oxygen Transport to Tissue, Springer US: Boston, MA, 1988,. pp. 183-189.

130. D.T. Delpy, M. Cope, P. van der Zee, S. Arridge, S. Wray, J. Wyatt, Estimation of optical pathlength through tissue from direct time of flight measurement, Phys Med Biol. 33 (1988) 1433-1442. 10.1088/0031-9155/33/12/008.

131. T. Talukdar, J.H. Moore, S.G. Diamond, Continuous correction of differential path length factor in near-infrared spectroscopy, J Biomed Opt. 18 (2013) 56001. 10.1117/1.Jbo.18.5.056001.

132. P.-H. Chou, T.-H. Lan, The role of near-infrared spectroscopy in Alzheimer's disease, Journal of Clinical Gerontology and Geriatrics. 4 (2013) 33-36. 10.1016/j.jcgg.2013.01.002.

133. P. Ekkekakis, Illuminating the black box: investigating prefrontal cortical hemodynamics during exercise with near-infrared spectroscopy, J Sport Exerc Psychol. 31 (2009) 505-553. 10.1123/jsep.31.4.505.

134. F. Scholkmann, M. Wolf, General equation for the differential pathlength factor of the frontal human head depending on wavelength and age, J Biomed Opt. 18 (2013) 105004. 10.1117/1.Jbo.18.10.105004.

135. G. Strangman, M.A. Franceschini, D.A. Boas, Factors affecting the accuracy of near-infrared spectroscopy concentration calculations for focal changes in oxygenation parameters, Neuroimage. 18 (2003) 865-879. 10.1016/s1053-8119(03)00021-1.

136. A. Duncan, J.H. Meek, M. Clemence, C.E. Elwell, P. Fallon, L. Tyszczuk, et al., Measurement of cranial optical path length as a function of age using phase resolved near infrared spectroscopy, Pediatr Res. 39 (1996) 889-894. 10.1203/00006450-199605000-00025. 
137. M. Essenpreis, C.E. Elwell, M. Cope, P. van der Zee, S.R. Arridge, D.T. Delpy, Spectral dependence of temporal point spread functions in human tissues, Appl Opt. 32 (1993) 418425. 10.1364/ao.32.000418.

138. K. Nakamura, K. Kurihara, H. Kawaguchi, T. Obata, H. Ito, E. Okada, Estimation of partial optical path length in the brain in subject-specific head models for near-infrared spectroscopy, Opt Rev. 23 ( 2016) 316-322. 10.1007/s10043-016-0179-9.

139. M. Izzetoglu, R. Holtzer, Effects of Processing Methods on fNIRS Signals Assessed During Active Walking Tasks in Older Adults, IEEE Trans Neural Syst Rehabil Eng. 12 (2020) http://dx.doi.org/10.1109/TNSRE.2020.2970407.

140. H.J. Niu, X. Li, Y.J. Chen, C. Ma, J.Y. Zhang, Z.J. Zhang, Reduced frontal activation during a working memory task in mild cognitive impairment: a non-invasive near-infrared spectroscopy study, CNS Neurosci Ther. 19 (2013) 125-131. 10.1111/cns.12046.

141. J.D. Schaeffer, A.S. Yennu, K.C. Gandy, F. Tian, H. Liu, H. Park, An fNIRS investigation of associative recognition in the prefrontal cortex with a rapid event-related design, $\mathrm{J}$ Neurosci Methods. 235 (2014) 308-315. 10.1016/j.jneumeth.2014.07.011.

142. F.B. Haeussinger, T. Dresler, S. Heinzel, M. Schecklmann, A.J. Fallgatter, A.C. Ehlis, Reconstructing functional near-infrared spectroscopy (fNIRS) signals impaired by extra-cranial confounds: an easy-to-use filter method, Neuroimage. 95 (2014) 69-79. 10.1016/j.neuroimage.2014.02.035.

143. T.J. Huppert, R.D. Hoge, S.G. Diamond, M.A. Franceschini, D.A. Boas, A temporal comparison of BOLD, ASL, and NIRS hemodynamic responses to motor stimuli in adult humans, Neuroimage. 29 (2006) 368-382. 10.1016/j.neuroimage.2005.08.065.

144. X. Cui, S. Bray, A.L. Reiss, Functional near infrared spectroscopy (NIRS) signal improvement based on negative correlation between oxygenated and deoxygenated hemoglobin dynamics, Neuroimage. 49 (2010) 3039-3046. 10.1016/j.neuroimage.2009.11.050. 
145. G. Allali, H.M. Blumen, H. Devanne, E. Pirondini, A. Delval, D. Van De Ville, Brain imaging of locomotion in neurological conditions, Neurophysiol Clin. 48 (2018) 337-359. http://dx.doi.org/10.1016/j.neucli.2018.10.004.

146. M. Ferrari, S. Bisconti, M. Spezialetti, S. Basso Moro, C. Di Palo, G. Placidi, et al., Prefrontal cortex activated bilaterally by a tilt board balance task: a functional near-infrared spectroscopy study in a semi-immersive virtual reality environment, Brain Topogr. 27 (2014) 353-365. 10.1007/s10548-013-0320-z.

147. D.J. Clark, D.K. Rose, S.A. Ring, E.C. Porges, Utilization of central nervous system resources for preparation and performance of complex walking tasks in older adults, Front Aging Neurosci. 6 (2014) http://dx.doi.org/10.3389/fnagi.2014.00217.

148. T. Huppert, J. Barker, B. Schmidt, S. Walls, A. Ghuman, Comparison of group-level, source localized activity for simultaneous functional near-infrared spectroscopymagnetoencephalography and simultaneous fNIRS-fMRI during parametric median nerve stimulation, Neurophotonics. 4 (2017) 015001. 10.1117/1.NPh.4.1.015001.

149. H. Sato, N. Yahata, T. Funane, R. Takizawa, T. Katura, H. Atsumori, et al., A NIRS-fMRI investigation of prefrontal cortex activity during a working memory task, Neuroimage. 83 (2013) 158-173. 10.1016/j.neuroimage.2013.06.043.

150. V. Scarapicchia, C. Brown, C. Mayo, J.R. Gawryluk, Functional Magnetic Resonance Imaging and Functional Near-Infrared Spectroscopy: Insights from Combined Recording Studies, Front Hum Neurosci. 11 (2017) 419. 10.3389/fnhum.2017.00419.

151. J.A. Noah, Y. Ono, Y. Nomoto, S. Shimada, A. Tachibana, X. Zhang, et al., fMRI Validation of fNIRS Measurements During a Naturalistic Task, J Vis Exp. (2015) e52116. 10.3791/52116.

152. A. Berger, F. Horst, F. Steinberg, F. Thomas, C. Muller-Eising, W.I. Schollhorn, et al., Increased gait variability during robot-assisted walking is accompanied by increased sensorimotor brain activity in healthy people, J Neuroeng Rehabil. 16 (2019) http://dx.doi.org/10.1186/s12984019-0636-3. 
153. M. Muthalib, A.R. Anwar, S. Perrey, M. Dat, A. Galka, S. Wolff, et al., Multimodal integration of fNIRS, fMRI and EEG neuroimaging, Clin Neurophysiol. 124 (2013) 2060-2062. 10.1016/j.clinph.2013.03.018.

154. R. Trevethan, Sensitivity, Specificity, and Predictive Values: Foundations, Pliabilities, and Pitfalls in Research and Practice, Front Public Health. 5 (2017) 307. 10.3389/fpubh.2017.00307.

155. S.J. Colcombe, A.F. Kramer, K.I. Erickson, P. Scalf, The implications of cortical recruitment and brain morphology for individual differences in inhibitory function in aging humans, Psychol Aging. 20 (2005) 363-375. 10.1037/0882-7974.20.3.363.

156. R. Cabeza, N.D. Anderson, J.K. Locantore, A.R. McIntosh, Aging gracefully: compensatory brain activity in high-performing older adults, Neuroimage. 17 (2002) 1394-1402. 10.1006/nimg.2002.1280.

157. P.A. Reuter-Lorenz, K.A. Cappell, Neurocognitive aging and the compensation hypothesis. , Curr Dir Psychol Sci. 17 (2008) 177-182.

158. Y. Bhambhani, R. Maikala, M. Farag, G. Rowland, Reliability of near-infrared spectroscopy measures of cerebral oxygenation and blood volume during handgrip exercise in nondisabled and traumatic brain-injured subjects, J Rehabil Res Dev. 43 (2006) 845-856. 10.1682/jrrd.2005.09.0151.

159. M.M. Plichta, M.J. Herrmann, C.G. Baehne, A.C. Ehlis, M.M. Richter, P. Pauli, et al., Eventrelated functional near-infrared spectroscopy (fNIRS): are the measurements reliable?, Neuroimage. 31 (2006) 116-124. 10.1016/j.neuroimage.2005.12.008.

160. D. Tsuzuki, I. Dan, Spatial registration for functional near-infrared spectroscopy: from channel position on the scalp to cortical location in individual and group analyses, Neuroimage. $85 \mathrm{Pt}$ 1 (2014) 92-103. 10.1016/j.neuroimage.2013.07.025.

161. S. Perrey, P. Besson, Studying brain activity in sports performance: Contributions and issues, Prog Brain Res. 240 (2018) 247-267. 10.1016/bs.pbr.2018.07.004. 
162. R.F. Rojas, X. Huang, K.-L. Ou, Region of Interest Detection and Evaluation in Functional near Infrared Spectroscopy, J. Near Infrared Spectrosc. 24 (2016) 317-326.

163. J.U. Blicher, C.J. Stagg, J. O'Shea, L. Ostergaard, B.J. Maclntosh, H. Johansen-Berg, et al., Visualization of altered neurovascular coupling in chronic stroke patients using multimodal functional MRI, J Cereb Blood Flow Metab. 32 (2012) 2044-2054. 10.1038/jcbfm.2012.105.

164. A.S. Salinet, N.C. Silva, J. Caldas, D.S. de Azevedo, M. de-Lima-Oliveira, R.C. Nogueira, et al., Impaired cerebral autoregulation and neurovascular coupling in middle cerebral artery stroke: Influence of severity?, J Cereb Blood Flow Metab. 39 (2019) 2277-2285. $10.1177 / 0271678 \times 18794835$.

165. L. Wang, H. Ayaz, M. Izzetoglu, Investigation of the source-detector separation in near infrared spectroscopy for healthy and clinical applications, J Biophotonics. 12 (2019) e201900175. 10.1002/jbio.201900175.

166. M.D. Fox, Mapping Symptoms to Brain Networks with the Human Connectome, N Engl J Med. 379 (2018) 2237-2245. 10.1056/NEJMra1706158.

167. B. Wang, M. Zhang, L. Bu, L. Xu, W. Wang, Z. Li, Posture-related changes in brain functional connectivity as assessed by wavelet phase coherence of NIRS signals in elderly subjects, Behav Brain Res. 312 (2016) 238-245. 10.1016/j.bbr.2016.06.037.

168. L.M. Hocke, I.K. Oni, C.C. Duszynski, A.V. Corrigan, B.D. Frederick, J.F. Dunn, Automated Processing of fNIRS Data-A Visual Guide to the Pitfalls and Consequences, Algorithms. 11 (2018) 10.3390/a11050067.

169. R. Holtzer, C. Schoen, E. Demetriou, J.R. Mahoney, M. Izzetoglu, C. Wanget al., Stress and gender effects on prefrontal cortex oxygenation levels assessed during single and dual-task walking conditions, Eur J Neurosci. 45 (2017) 660-670. 10.1111/ejn.13518.

170. R. Holtzer, J. Verghese, G. Allali, M. Izzetoglu, C. Wang, J.R. Mahoney, Neurological Gait Abnormalities Moderate the Functional Brain Signature of the Posture First Hypothesis, Brain Topogr. 29 (2016) 334-343. 10.1007/s10548-015-0465-z. 
171. R. Holtzer, J. Yuan, J. Verghese, J.R. Mahoney, M. Izzetoglu, C. Wang, Interactions of Subjective and Objective Measures of Fatigue Defined in the Context of Brain Control of Locomotion, J Gerontol A Biol Sci Med Sci. 72 (2017) 417-423. 10.1093/gerona/glw167.

172. M. Lucas, M.E. Wagshul, M. Izzetoglu, R. Holtzer, Moderating Effect of White Matter Integrity on Brain Activation During Dual-Task Walking in Older Adults, J Gerontol A Biol Sci Med Sci. 74 (2019) 435-441. http://dx.doi.org/10.1093/gerona/gly131.

173. R. Holtzer, R. Kraut, M. Izzetoglu, K. Ye, The effect of fear of falling on prefrontal cortex activation and efficiency during walking in older adults, GeroScience. 41 (2019) 89-100. http://dx.doi.org/10.1007/s11357-019-00056-4.

174. X.N. Zuo, J.S. Anderson, P. Bellec, R.M. Birn, B.B. Biswal, J. Blautzik, et al., An open science resource for establishing reliability and reproducibility in functional connectomics, Sci Data. 1 (2014) 140049. 10.1038/sdata.2014.49.

175. C.L. Tardif, A. Schafer, R. Trampel, A. Villringer, R. Turner, P.L. Bazin, Open Science CBS Neuroimaging Repository: Sharing ultra-high-field MR images of the brain, Neuroimage. 124 (2016) 1143-1148. 10.1016/j.neuroimage.2015.08.042. 


\section{TABLES}

Table 1. Summary of key point recommendations and considerations 


\section{FIGURES}

Figure 1. Examples of block design (A) and event-related design (B) used in fNIRS studies of posture and gait. The interval of reference distinguishes between designs.

A) Block design: the concentration in oxygenated haemoglobin ( $\mathrm{HbO2}$ ) during a balance / gait task (0s to 20 s, here) is normalised to a static baseline (-10 to 0 s, here) immediately preceding the onset of the task of interest. The zero crossing indicates the start of the actual task condition (adapted from Mirelman et al., 2014) [9].

B) Event-related design: the concentration in oxygenated haemoglobin ( $\mathrm{HbO2}$ ) during an "event", for example, a turn (blue trace) or a freezing of gait (FOG) event as displayed here, is normalised to a dynamic baseline, here normal walking (green trace) (adapted from Maidan et al., 2015) [32].

Figure 2. Summary of fNIRS data processing steps.

Figure 3. Examples of different levels of filtering on $\mathrm{HbO2}$ signal acquired from prefrontal cortex channels during: (A) 20 stepping trials of inhibitory stepping test; (B) walking. Note how the addition of other filters (wavelet with or without CBSI filters) attenuates the signal. 
SUPPLEMENTARY MATERIALS

Table S1. Checklist of items to consider at processing and reporting steps of fNIRS data collected in studies of posture and gait. 